\title{
LA-UR-21-23851
}

Approved for public release; distribution is unlimited.

Title: $\quad$ Modeling the Fission Meter

Author(s): $\quad$ Marcath, Matthew James

Mayo, Douglas R.

Bates, Cameron Russell

Intended for: Report

Issued: 
Disclaimer:

Los Alamos National Laboratory, an affirmative action/equal opportunity employer, is operated by Triad National Security, LLC for the National Nuclear Security Administration of U.S. Department of Energy under contract 89233218CNA000001. By approving this article, the publisher recognizes that the U.S. Government retains nonexclusive, royalty-free license to publish or reproduce the published form of this contribution, or to allow others to do so, for U.S. Government purposes. Los Alamos National Laboratory requests that the publisher identify this article as work performed under the auspices of the U.S. Department of Energy. Los Alamos National Laboratory strongly supports academic freedom and a researcher's right to publish; as an institution, however, the Laboratory does not endorse the viewpoint of a publication or guarantee its technical correctness. 


\section{Modeling the Fission Meter}

Matthew J. Marcath, Douglas R. Mayo, and Cameron R. Bates

31 March 2021 


\section{Contents}

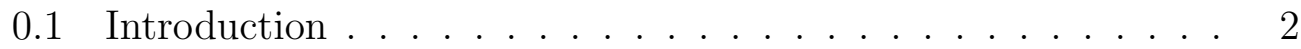

0.2 Python script: ptrac_feynman_y.py . . . . . . . . . . . . 14

0.3 $\quad$ MCNP6 model: Cf-252 point source . . . . . . . . . . . 26

0.4 Python script configuration: Cf-252 point source . . . . . . . 32

0.5 MCNP6 model: TAI 1 . . . . . . . . . . . . . . . . . 34

0.6 Python script configuration: TAI $1 \ldots \ldots \ldots$

0.7 Python results plotter $\ldots \ldots \ldots \ldots$. . . . . . . . . . . . 42 


\subsection{Introduction}

This presentation includes an overview of the process, example inputs, and example results for the Fission Meter and its response. 


\section{(A) Los Alamos

\section{Modeling the Fission Meter}

Matthew J. Marcath, Douglas R. Mayo, and Cameron R. Bates

6 April 2021 


\section{Fission Meter: MCNP model and Feynman-Y}

- Fission Meter is modeled in MCNP.

- MCNP model produces a PTRAC file of He-3 captures.

- Python script reads the PTRAC file and produces Feynman-Y results.

- Software you need:

- MCNP6 - preferably LANL's development version with HDF5.

- Python 3.7 or greater with SciPy

- MCNPTools - preferably 5.2.0 for HDF5

- All software is or can be made available on LANL HPC

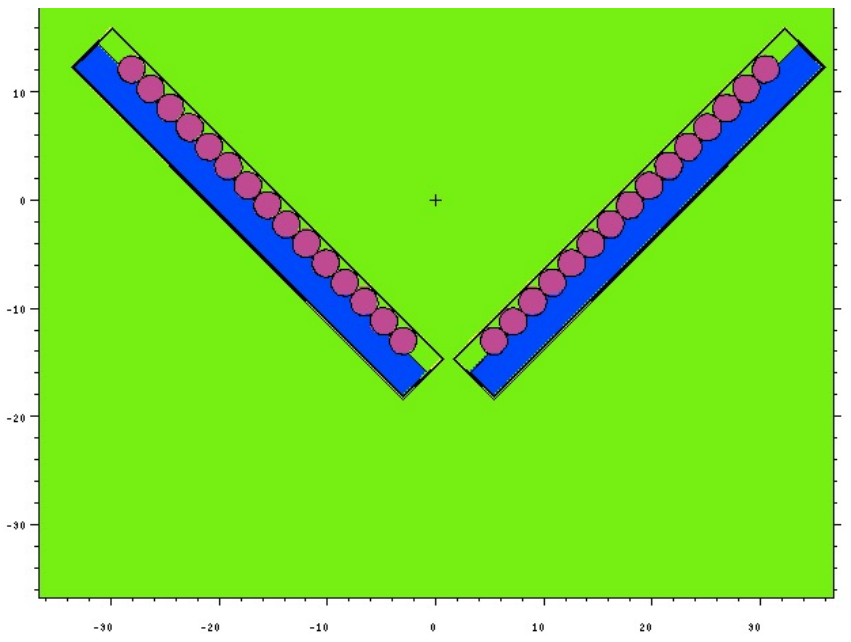

- Example contains:

- fm_Cf.i and fm_TAI.i - MCNP input

- ptrac_feynman_y.py - analysis script

- fy_config.ini - analysis script configuration

- plot_n_coinc.py - results plotter

- Outputs 


\section{Work flow}

- MCNP writes a ptrac file containing events with He-3 captures in the tube's active volumes.

- Python script

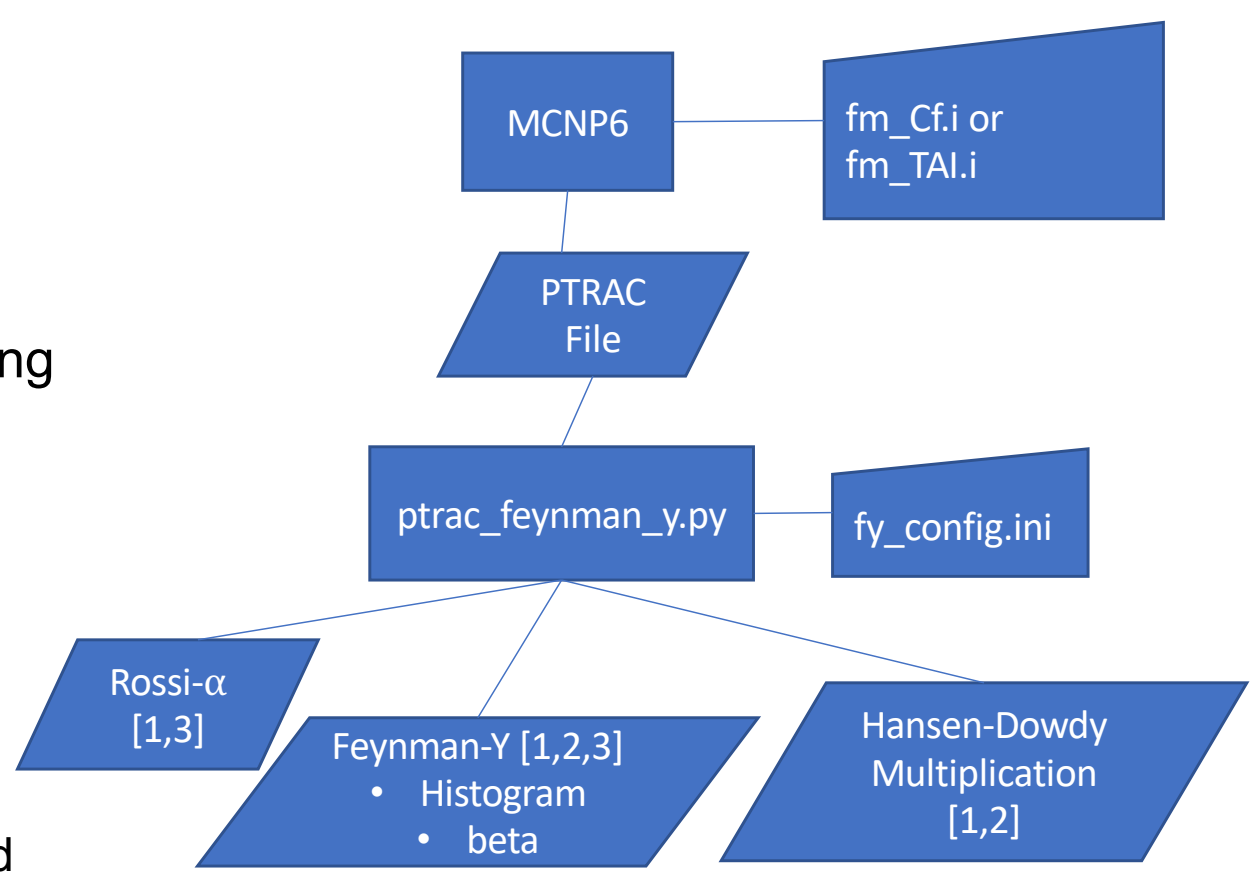

- Time distributes MCNP histories

- Applies dead time.

- Makes Rossi- $\alpha$ histogram and dieaway fit.

- Makes Feynman-Y histograms and Hansen-Dowdy multiplication estimates.

1. Smith-Nelson, M., et al., "Neutron Specialist Handbook and Informational Text (Unclassified Version)", LA-UR-07-6170, 2007.

2. Dowdy, E J, Hansen, G E, \& Robba, A A. "Feynmann variance-to-mean method." United States, 1985.

3. Ensslin, N., "Passive Nondestructive Assay of Nuclear Materials: Principles of Neutron Coincidence Counting." LA-UR-90-732, 1991. 


\section{Analysis script config: Cf-252 point source}

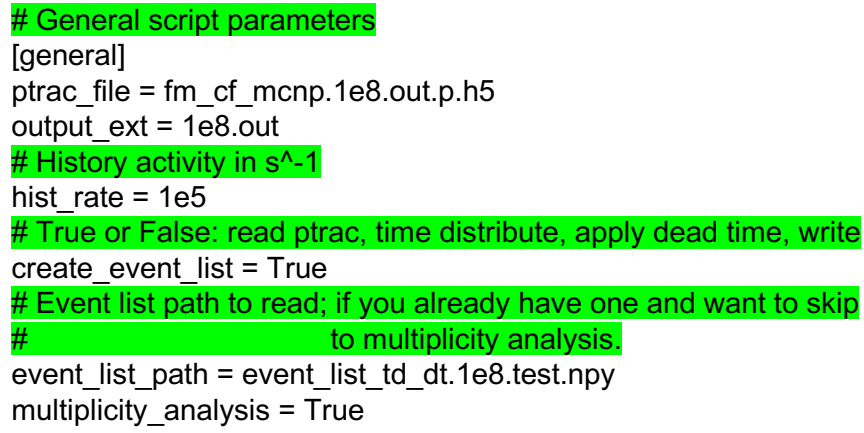

\section{\# system and material parameters}

[system]

\# Uncorrelated neutron rate in [n/s] i.e. (alpha,n)

S_0 $=0$

\# Rossi alpha histogram range [us]

rossi_max_time $=100$

rossi_bin_step $=1$

rossi_accidental_start $=75$

rossi_fit_start $=\overline{5}$

rossi_fit_end $=50$

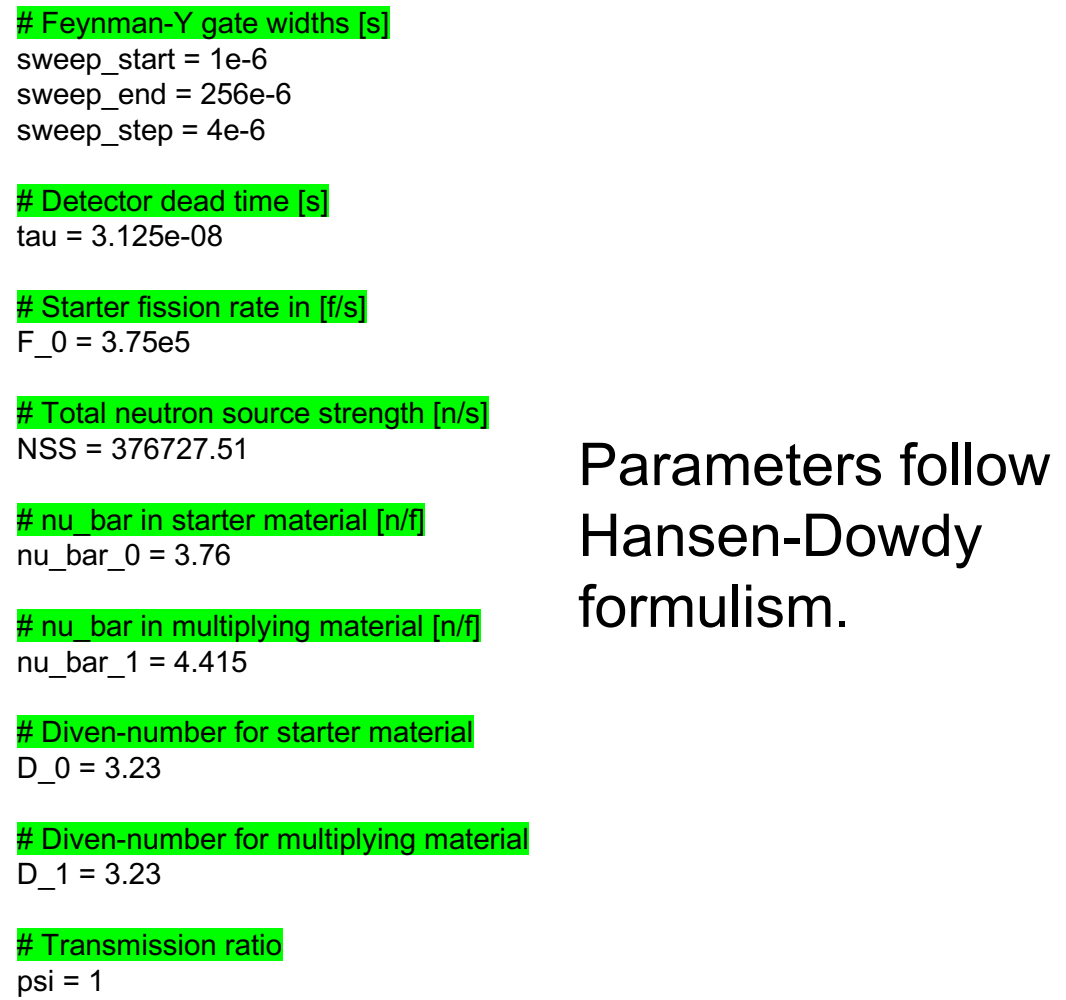

Parameters follow Hansen-Dowdy formulism. 


\section{Results: Cf-252 point source}

- Results and fits are printed to text file.

- Results saved to NumPy files

- Event list

- Rossi- $\alpha$

- Feynman-Y multiplicity

- Feynman-Y $\beta$

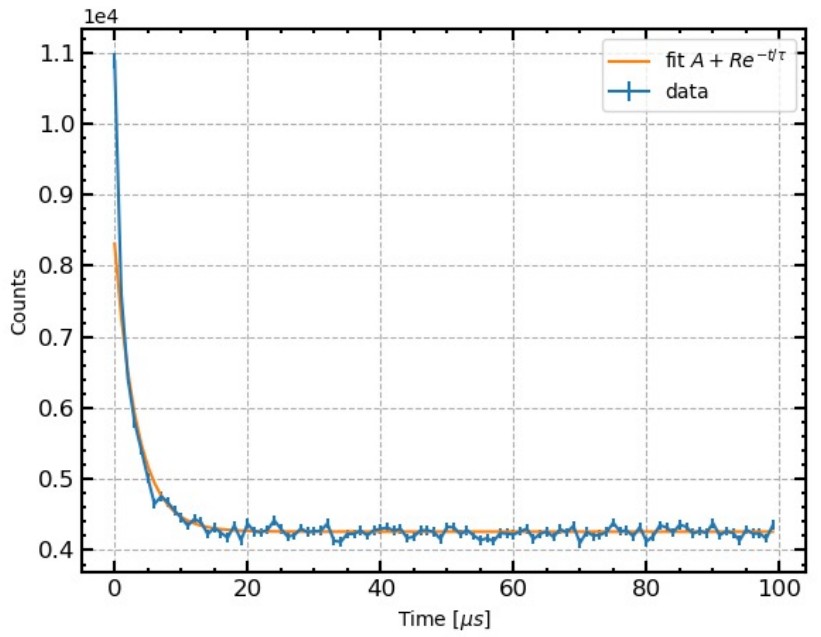

$\mathrm{A}=4.26 \mathrm{E}+03 \mathrm{R}=4.05 \mathrm{E}+03 \quad \tau=3.4$ us 


\section{Results: Cf-252 point source}
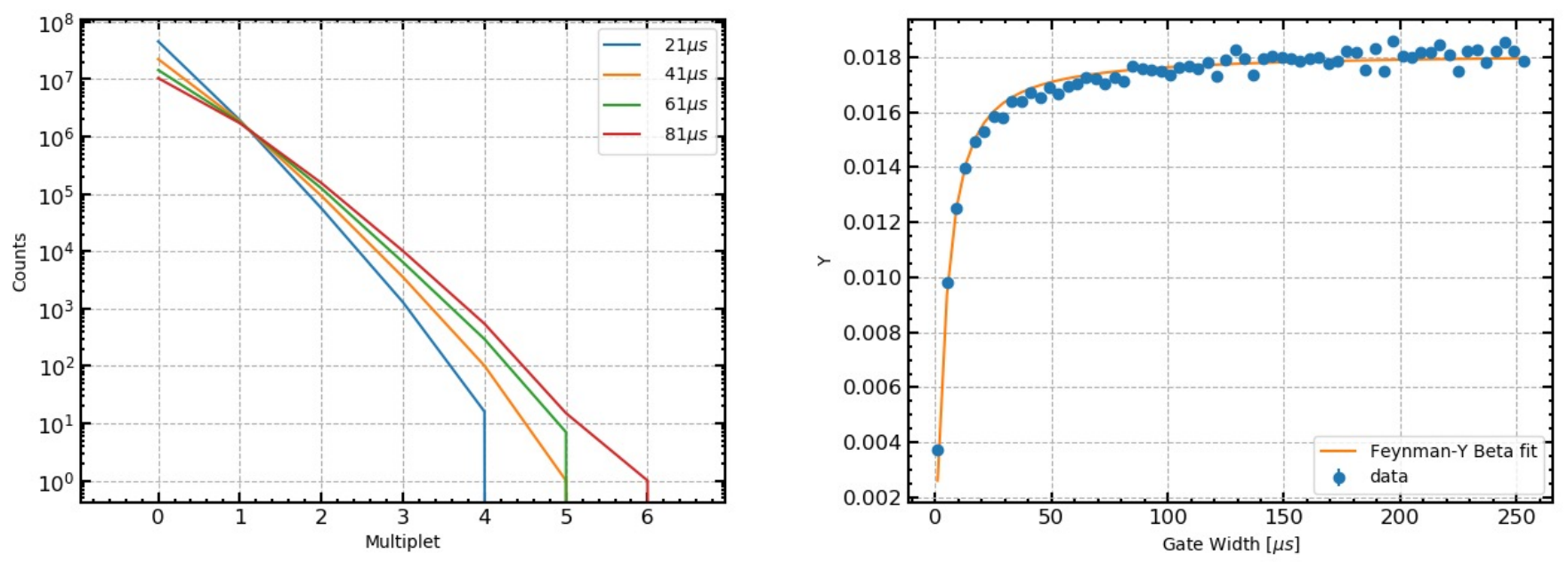

$K=1.8 E-02 \beta=3.4 E+05 \mathrm{~s}^{-1}$ 
MCNP Model: Type 1 TAl
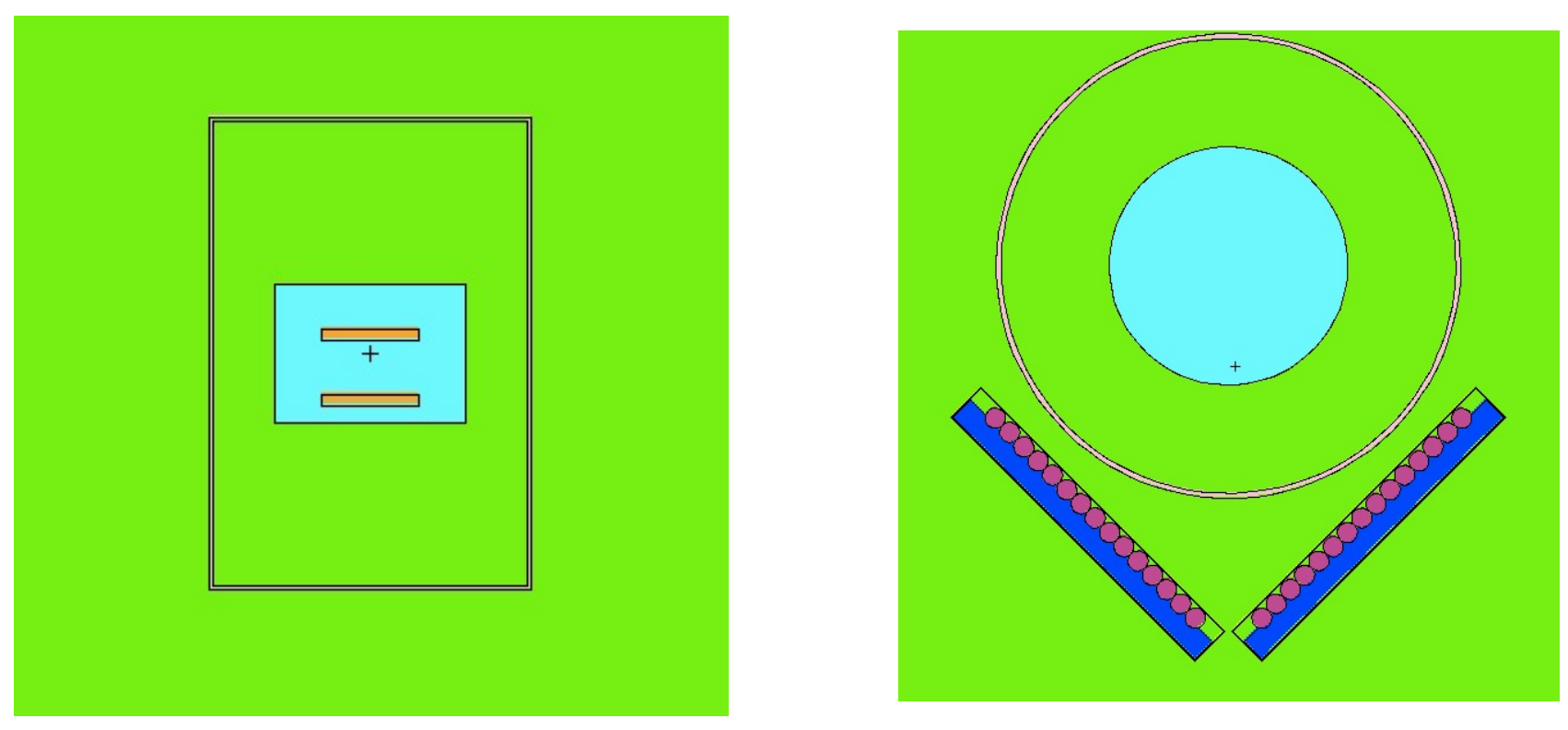


\section{Analysis script config: Type 1 TAl}

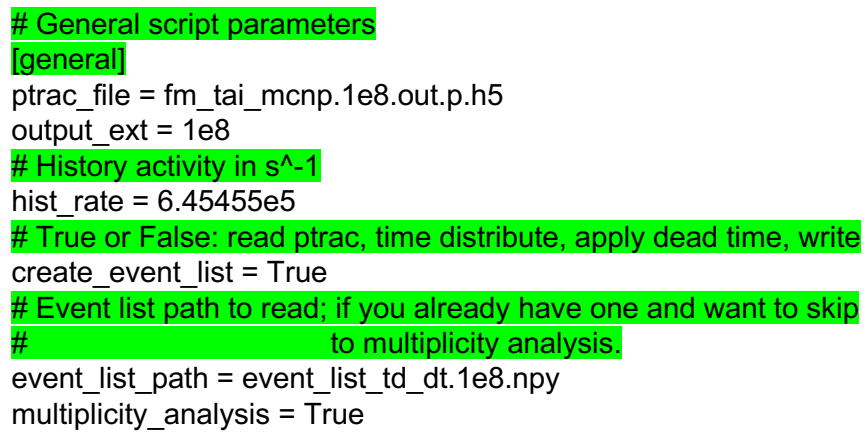

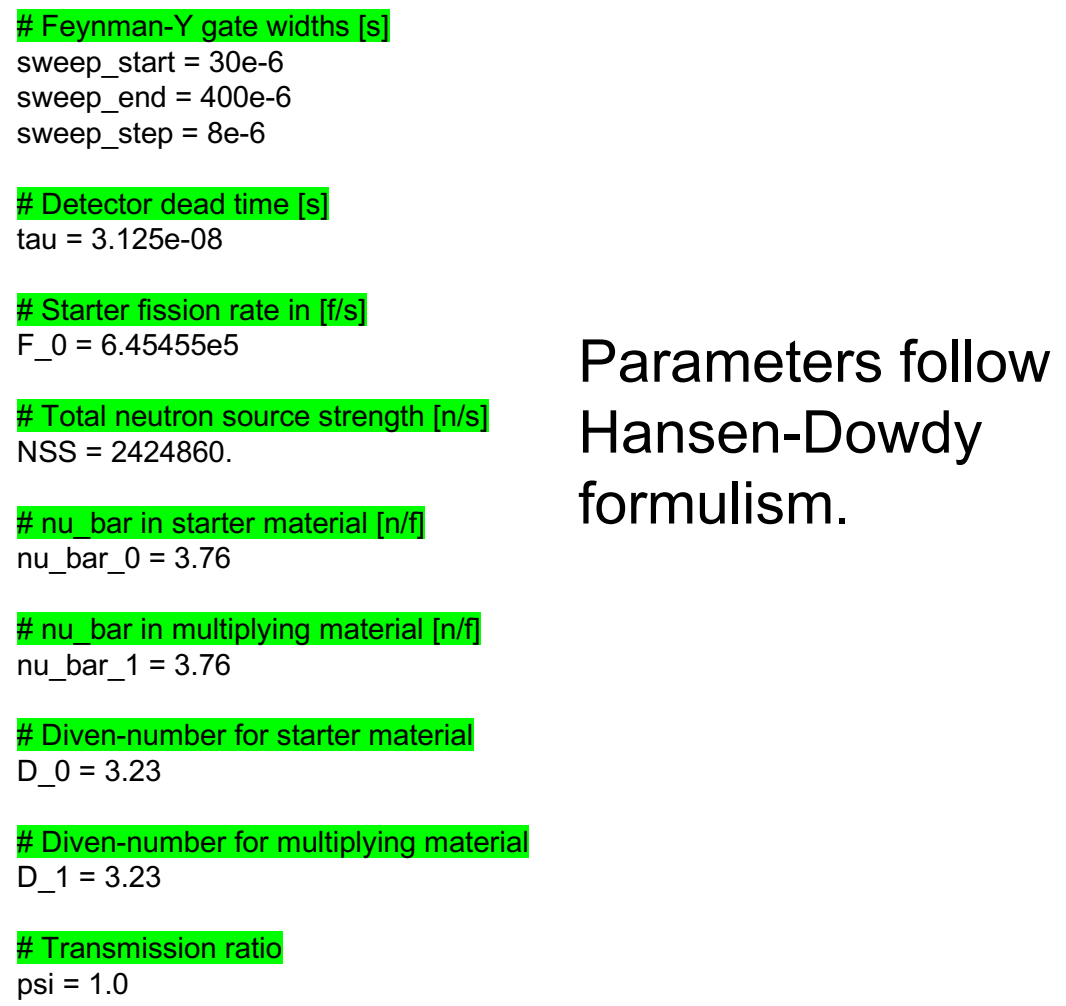

\section{Parameters follow Hansen-Dowdy formulism.}




\section{Results: Type 1 TAl}

- Results and fits are printed to text file.

- Results saved to NumPy files

- Event list

- Rossi- $\alpha$

- Feynman-Y multiplicity

- Feynman-Y $\beta$

- Bad multiplication estimate $p_{f}<<p_{c}$

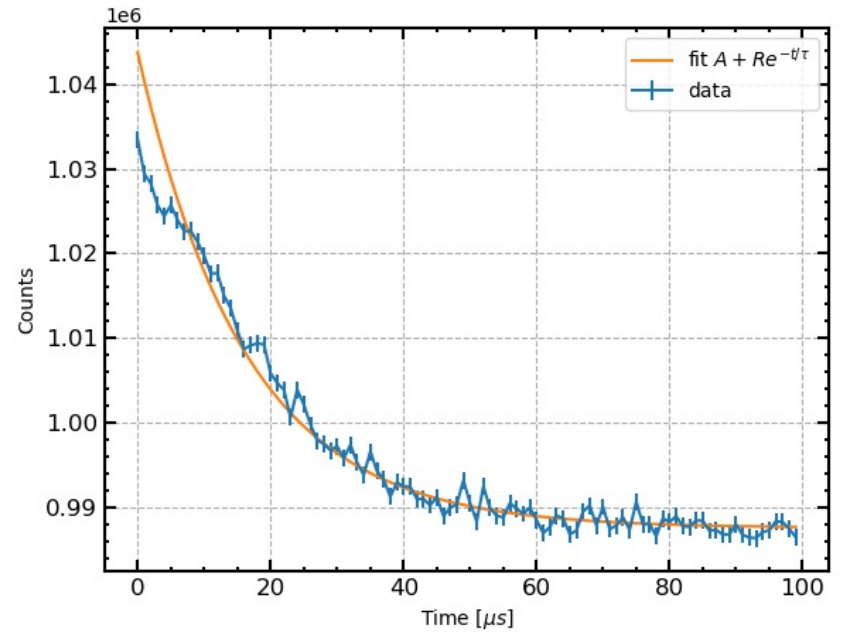

$\mathrm{A}=9.88 \mathrm{E}+05 \mathrm{R}=5.61 \mathrm{E}+04 \tau=16$ us 


\section{Results: Type 1 TAl}
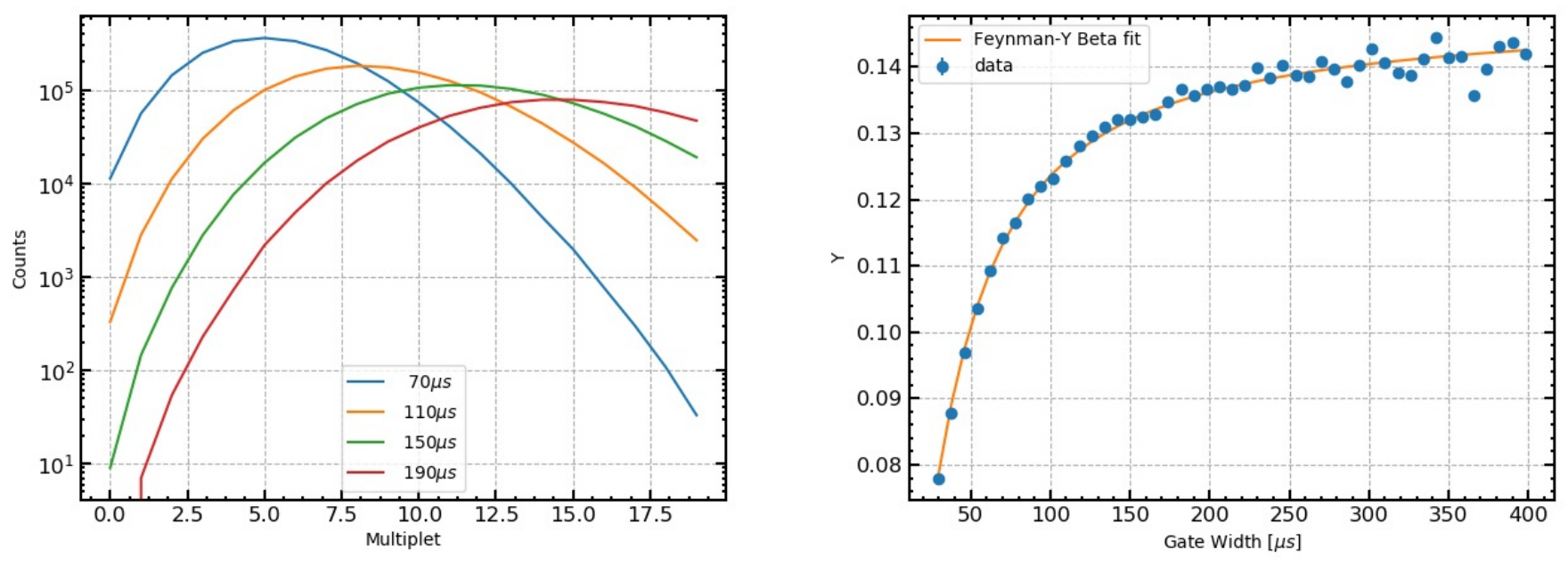

$K=1.49 E-01 \beta=5.87 E+04 s^{-1}$ 


\section{Q. Los Alamos}




\subsection{Python script: ptrac_feynman_y.py}

Given a configuration file, the python script processes MCNP PTRAC outputs to produce Rossi-alpha and Feynman-Y histograms.

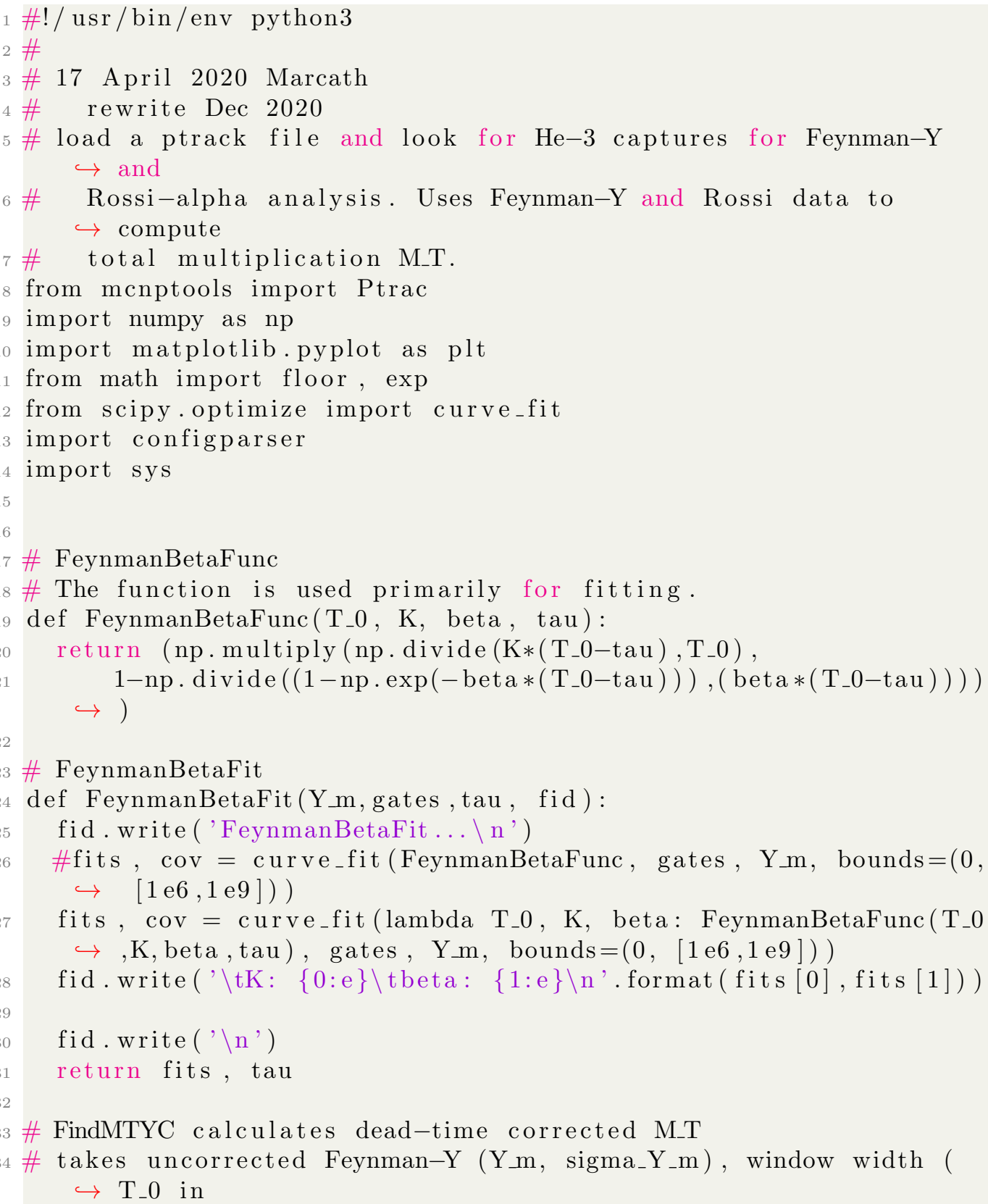




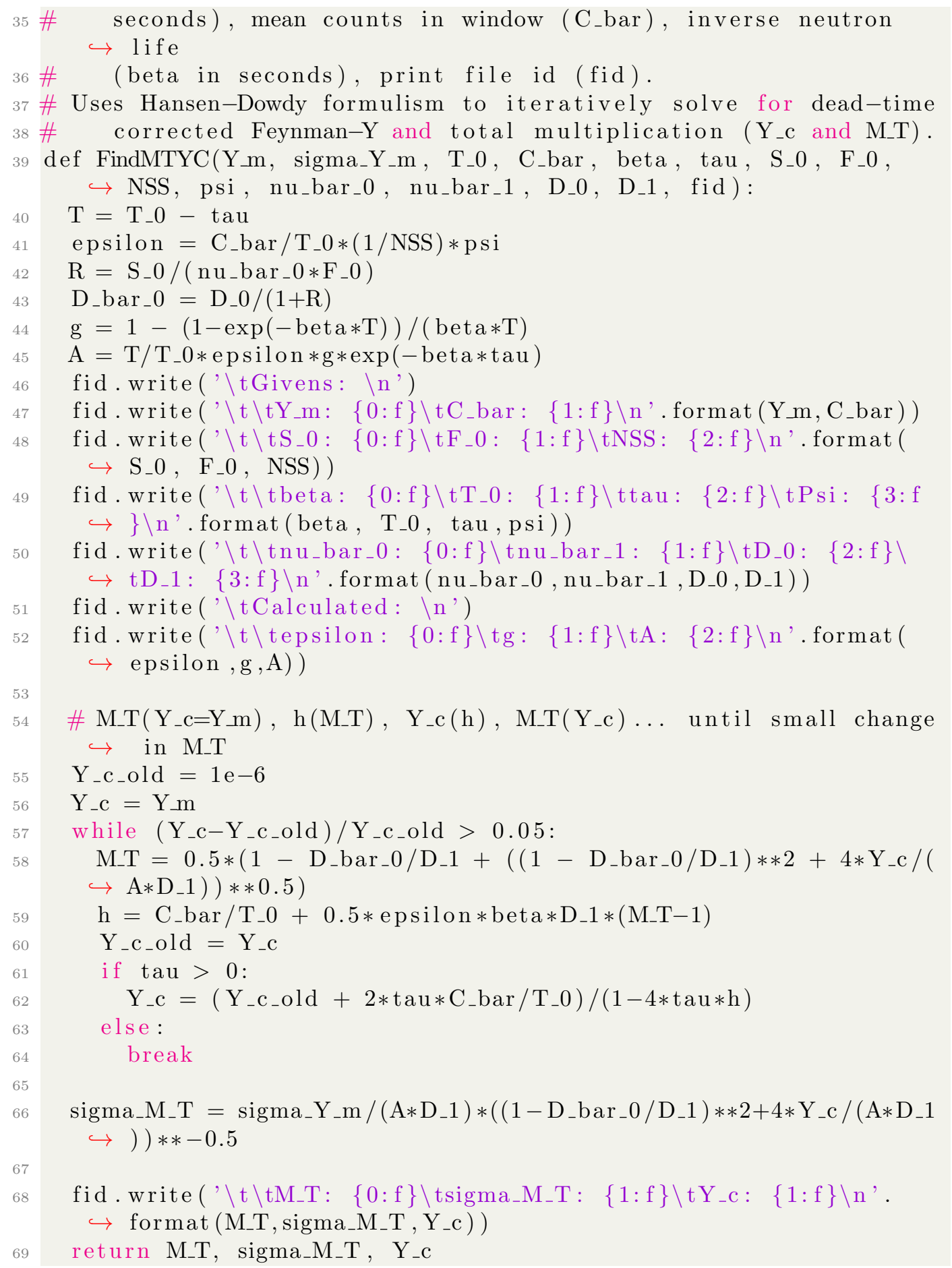




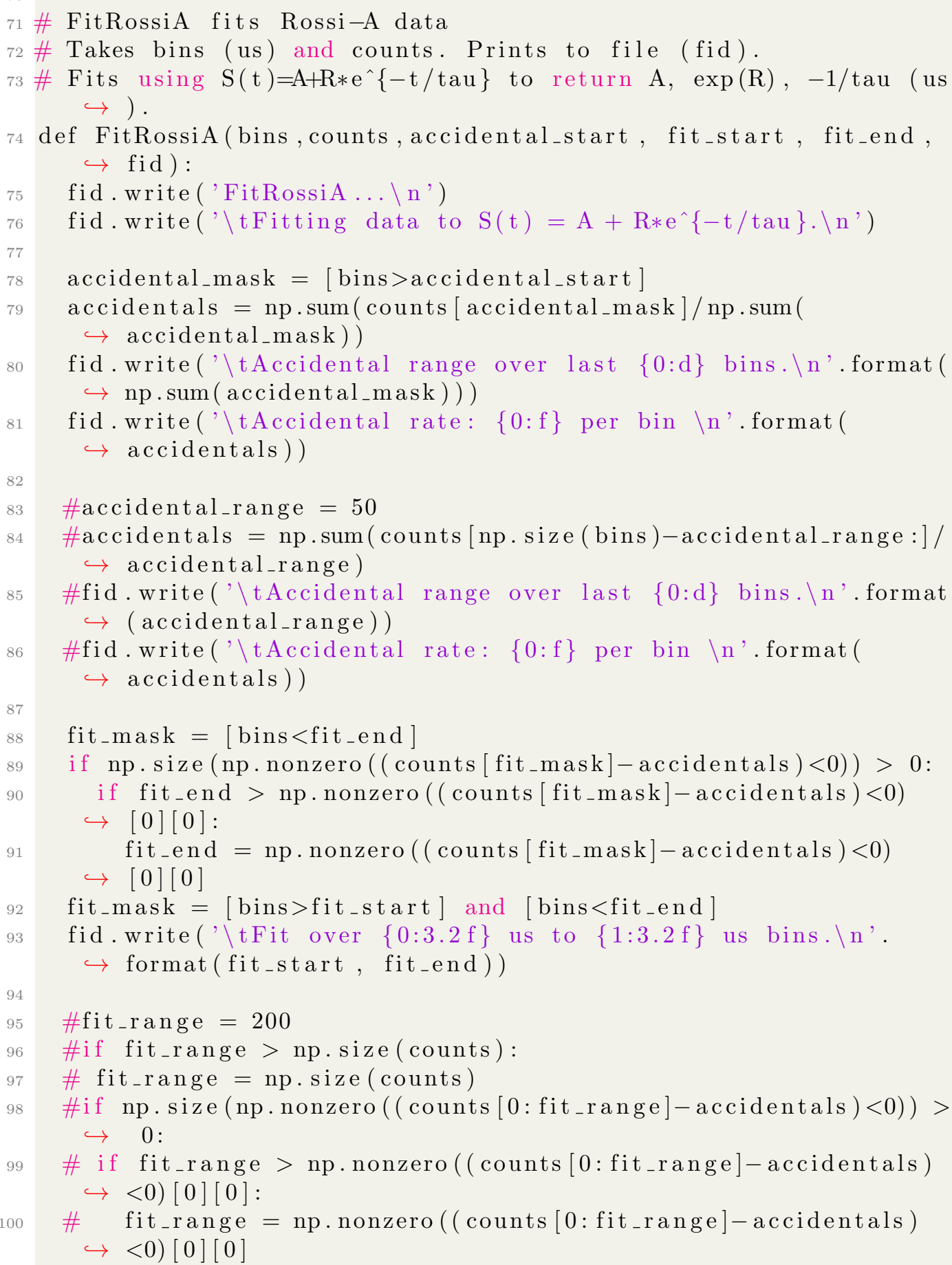


\#fid.write ('\tFit over the first $\{0: d\}$ bins. $\backslash n$ '. format (

$\hookrightarrow$ fit_range))

fit $=$ np.polyfit (bins [fit_mask], np. log (counts [fit_mask]-

$\hookrightarrow$ accidentals), 1 )

$\#$ fit $=$ np.polyfit(bins [0:fit_range], np. log(counts [0:fit_range $\hookrightarrow$ ]-accidentals $), 1)$

fid. write ('\t1/tau: $\{0: \mathrm{f}\}$ us $\backslash \mathrm{tR}:\{1: \mathrm{f}\} \backslash \mathrm{n}$ '. format $(-1.0 / \mathrm{fit}[0]$, $\hookrightarrow \exp ($ fit $[1])))$

fid. write ('\n')

return accidentals, $-1.0 /$ fit $[0], \exp ($ fit $[1])$

\# RossiA histograms capture data from event_list.

\# Takes event_list (must have ['time'] column) and histograms $\hookrightarrow$ with MAX TIME window. Prints data to file (fid).

* Returns histogram bins and counts.

def Rossia(event_list, MAX_TIME, bin_width, fid):

fid.write ('RossiA ...\n')

\#MAX_TIME $=50$ \# microseconds

\#bin_width $=0.2 \quad \#$ microseconds

fid.write ('\tMaximum time: $\{0: \mathrm{f}\}$ us $\backslash \mathrm{n}$ '. format (MAX_TIME))

fid.write (' $\backslash$ tBin width: $\{0: f\}$ us $\backslash n$ '. format (bin_width))

bins $=$ np.arange $(0$, MAXTIME, bin_width $)$

counts $=$ np.zeros $((n p \cdot \operatorname{size}($ bins $)))$

MAXEVENTS $=$ np.size ( event_list ['time'])

for i, time_i in enumerate(event_list ['time']) :

$$
j=i+1
$$

time $=$ event_list ['time'] $[j]$

if (time+MAXTIME $* 1.0$ e-6 $>$ event_list [ 'time'] [MAX EVENTS-1] or $\mathrm{j}>$ MAXEVENTS) : break

while (time $<$ time_i+MAXTIME $* 1.0 \mathrm{e}-6$ and $\mathrm{j}<$ MAXEVENTS) : bin_num $=$ floor $(($ time - time_i $) * 1.0 \mathrm{e} 6 /$ bin_width $)$ counts [bin_num] $+=1$

$\mathrm{j}+=1$

time $=$ event_list ['time'][j]

fid.write('\tRossi alpha histogram \n') 


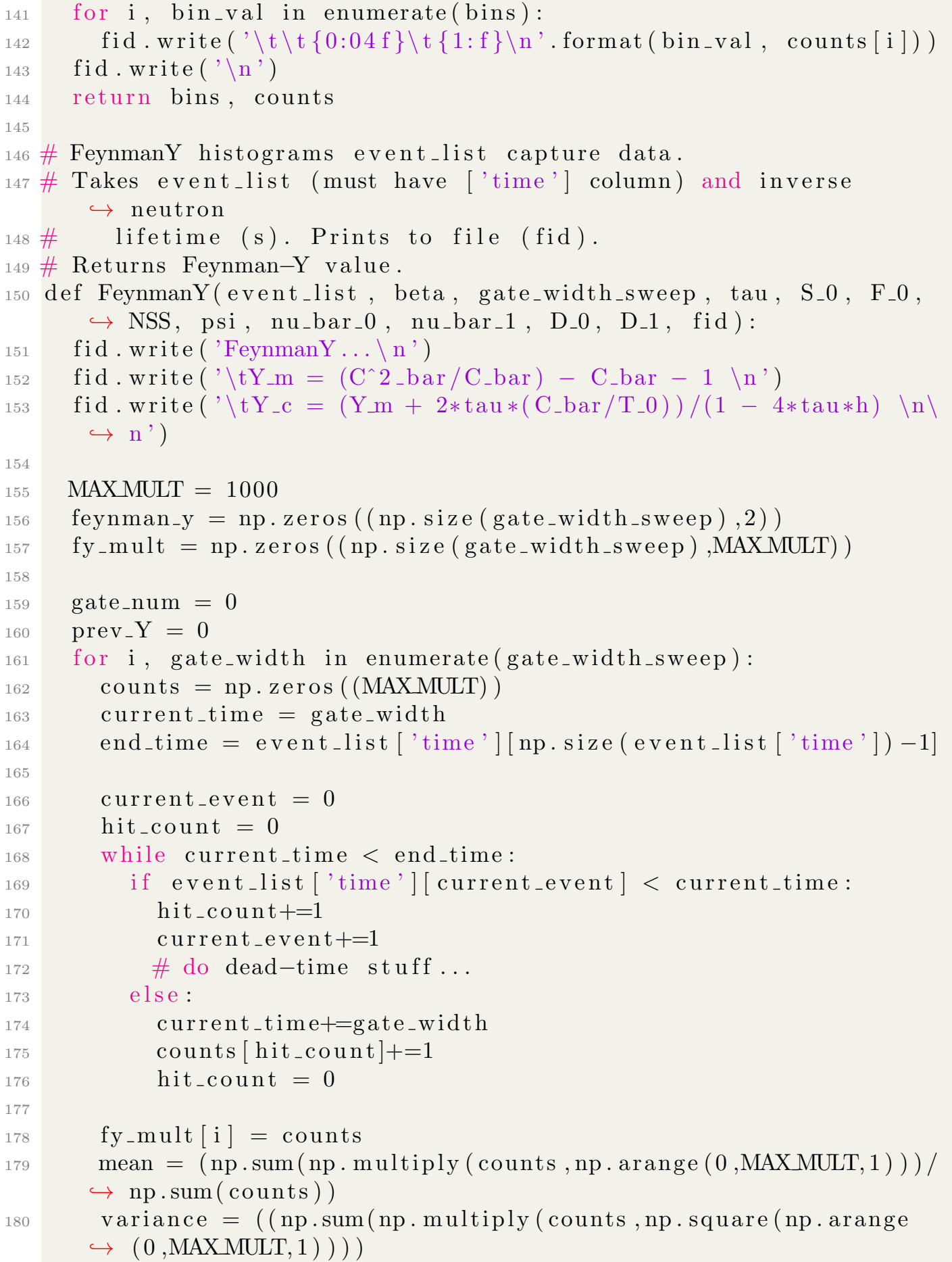




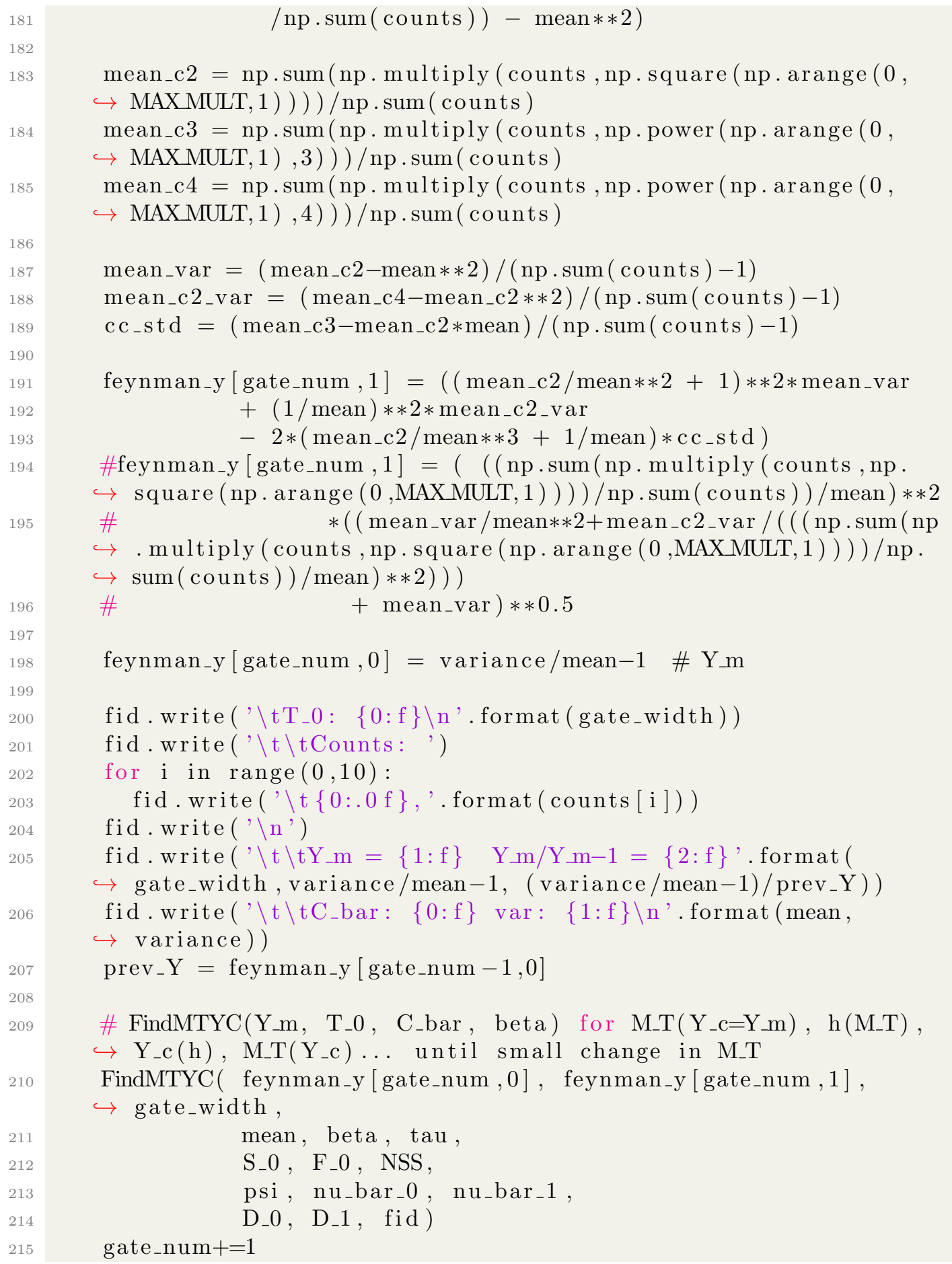




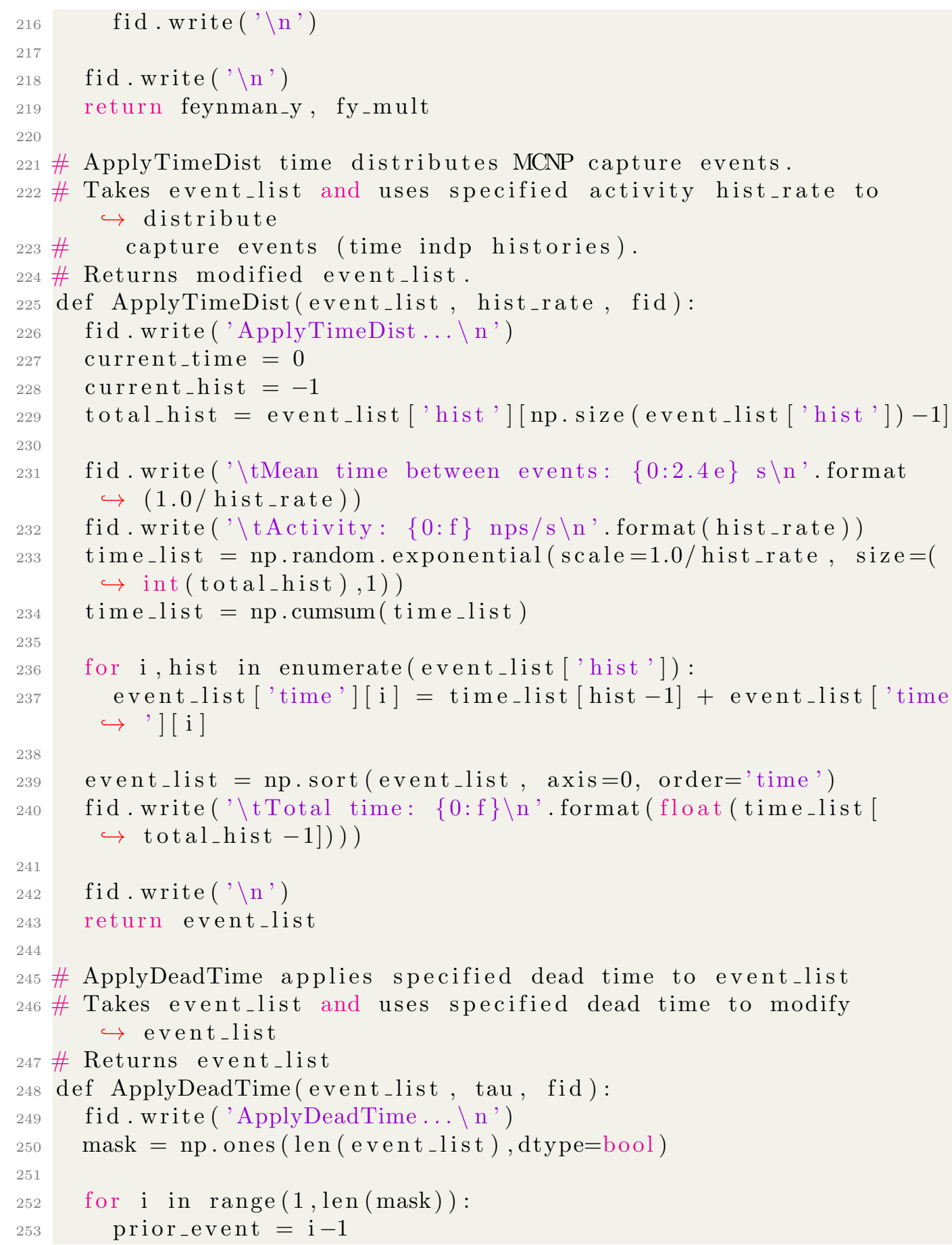

244

$$
\text { fid.write ('\n') }
$$

fid.write ('\n')

return feynman_y, fy_mult

\# ApplyTimeDist time distributes MCNP capture events.

\# Takes event_list and uses specified activity hist_rate to $\hookrightarrow$ distribute

\# capture events (time indp histories).

\# Returns modified event_list.

def ApplyTimeDist(event_list, hist_rate, fid):

fid .write ('ApplyTimeDist...\n')

current_time $=0$

current_hist $=-1$

total_hist $=$ event_list ['hist'] [np.size( event_list ['hist'] $)-1]$

fid.write(' $\backslash$ tMean time between events: $\{0: 2.4 \mathrm{e}\} \quad \mathrm{s} \backslash \mathrm{n}$ '. format $\hookrightarrow(1.0 /$ hist_rate $))$

fid.write (' $\backslash$ tActivity: $\{0: \mathrm{f}\} \mathrm{nps} / \mathrm{s} \backslash \mathrm{n}$ '. format (hist_rate))

time_list $=n p \cdot$ random. exponential $($ scale $=1.0 /$ hist_rate, size $=($ $\hookrightarrow$ int (total_hist), 1))

time_list $=$ np.cumsum(time_list)

for i, hist in enumerate( event_list ['hist']): event_list ['time'][i] = time_list [hist -1$]+$ event_list ['time $\hookrightarrow$ ' ] [ i ]

event_list $=$ np.sort (event_list, axis $=0$, order='time') fid.write (' $\backslash$ tTotal time: $\{0: \mathrm{f}\} \backslash \mathrm{n}$ '. format (float (time_list [ $\hookrightarrow$ total_hist -1$]))$ )

fid.write ('\n')

return event_list

\# ApplyDeadTime applies specified dead time to event_list

\# Takes event_list and uses specified dead time to modify $\hookrightarrow$ event_list

\# Returns event_list

def ApplyDeadTime(event_list, tau, fid):

fid .write ('ApplyDeadTime...\n')

mask $=$ np.ones $($ len $($ event_list $)$, dtype=bool $)$

for i in range $(1$, len (mask)) :

prior_event $=\mathrm{i}-1$ 


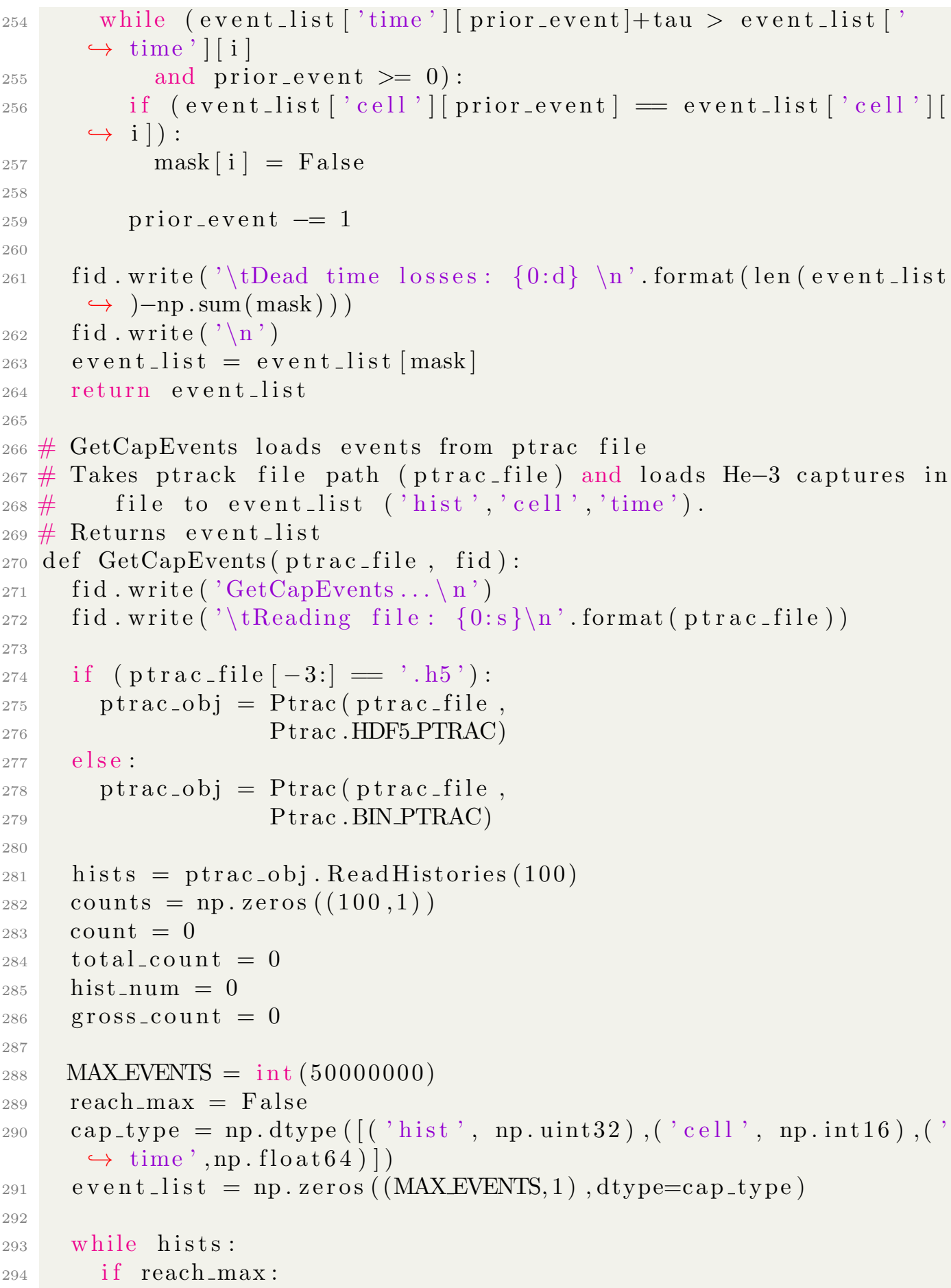




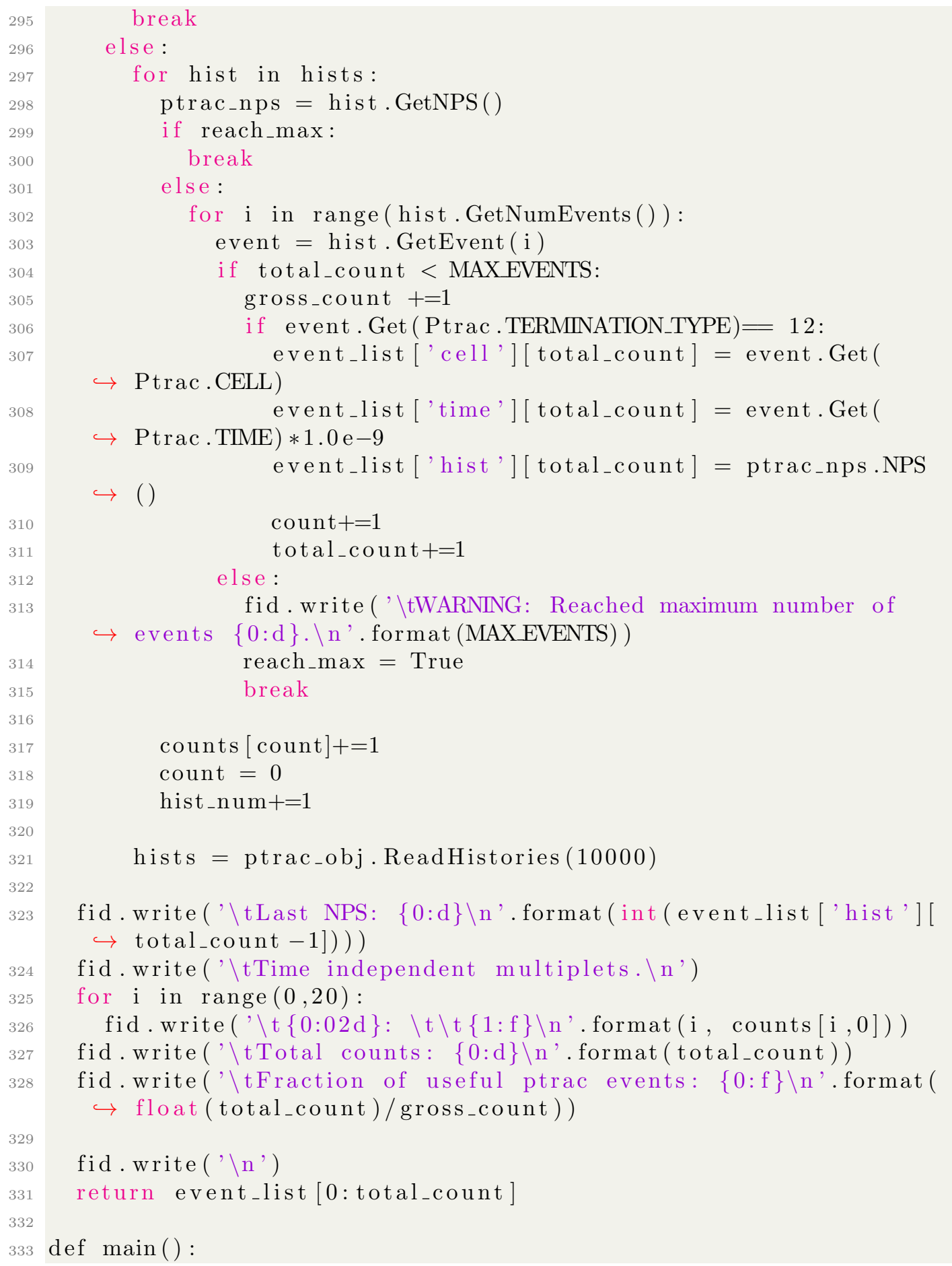


\# Read the configuration file and pull out relevant parameters $\hookrightarrow$.

\# General script parameters here.

config_file = 'config.ini'

if $(\operatorname{len}(\operatorname{sys} \cdot \operatorname{argv})>1)$ :

config-file $=\operatorname{str}(\operatorname{sys} \cdot \operatorname{argv}[1])$

config $=$ configparser. ConfigParser ()

config.read (config_file)

file_name = config ['general'] ['ptrac_file']

out_file_extension = config['general'] ['output_ext']

hist_rate $=$ float (config ['general'] ['hist_rate'])

out_file_name $=$ 'FY_analysis. $\{$ ext $\}$.txt'. format $($ ext $=$ $\hookrightarrow$ out_file_extension)

create_event_list $=$ (config['general'] ['create_event_list'] $=$ $\hookrightarrow$ 'True')

event_list_path $=$ config['general']['event_list_path']

multiplicity_analysis $=$ ( config ['general'] ['

$\hookrightarrow$ multiplicity_analysis' $]=$ 'True')

\# Multiplicity analysis parameters here.

rossi_max_time $=$ float (config['system'] ['rossi_max_time'])

rossi_bin_step = float (config['system']['rossi_bin_step'])

rossi_accidental_start = float (config ['system'] ['

$\hookrightarrow$ rosisicacidental_start' $])$

rossi_fit_start $=$ float (config['system'] ['rossi_fit_start'])

rossi_fit_end = float(config['system'] ['rossi_fit_end'])

rossi_bin_step = float( config['system']['rossi_bin_step'])

$\operatorname{tau}=$ float (config['system']['tau'])

$\mathrm{S}_{-} 0=$ float (config ['system'] ['S_0'])

$\mathrm{F}_{-} 0=$ float (config ['system'] ['F_0'])

gate_width_sweep $=$ np. arange( float (config ['system'] ['

$\hookrightarrow$ sweep_start']),

$\hookrightarrow$ sweep_end']),

$\hookrightarrow$ sweep_step']))

float (config ['system'] ['

float ( config ['system'] ['

NSS $=$ float ( config ['system'] [ 'NSS'])

nu_bar_0 = float (config ['system'] ['nu_bar_0'])

nu_bar_1 = float (config ['system']['nu_bar_1'])

D_0 $=$ float (config ['system'] ['D_0']) 


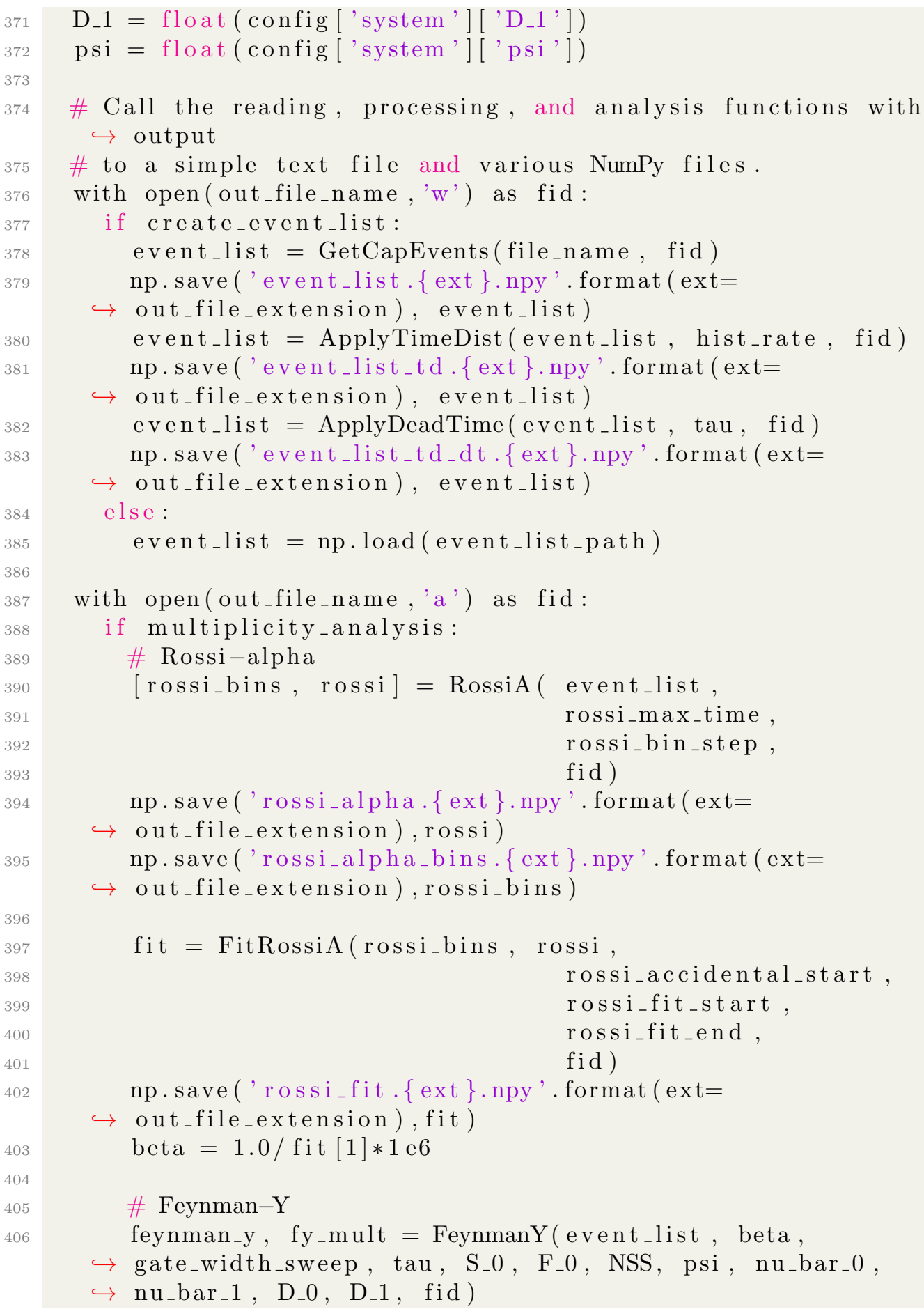




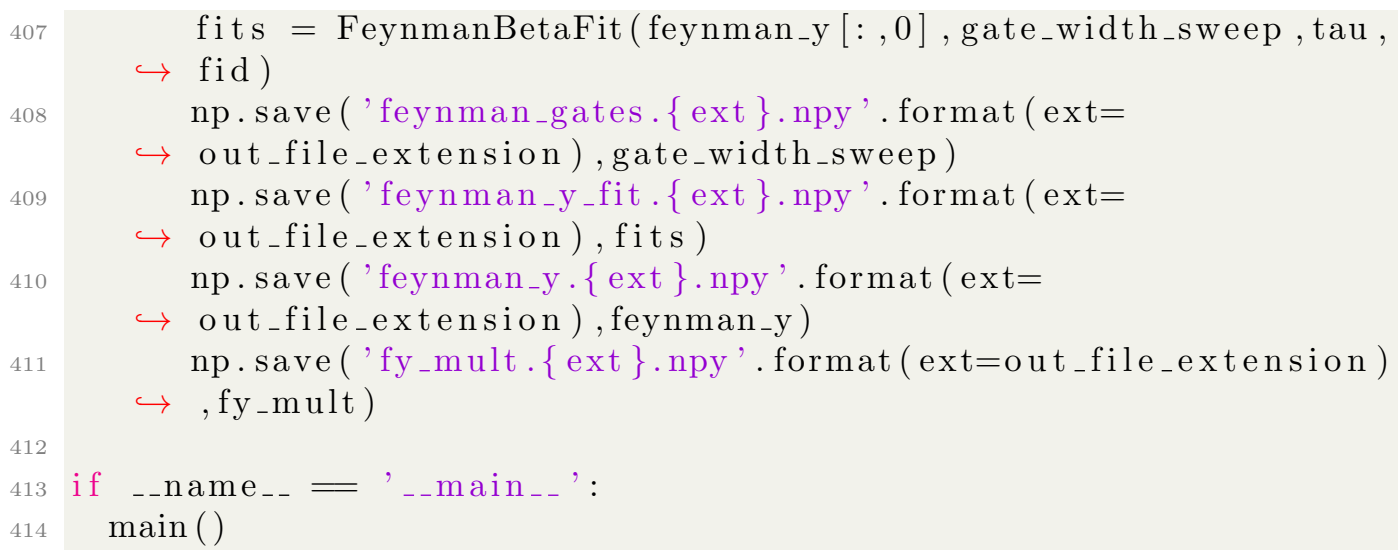




\subsection{MCNP6 model: Cf-252 point source}

The MCNP model describes a neutron measurement with the Fission Meter of a point Cf-252 spontaneous fission source.

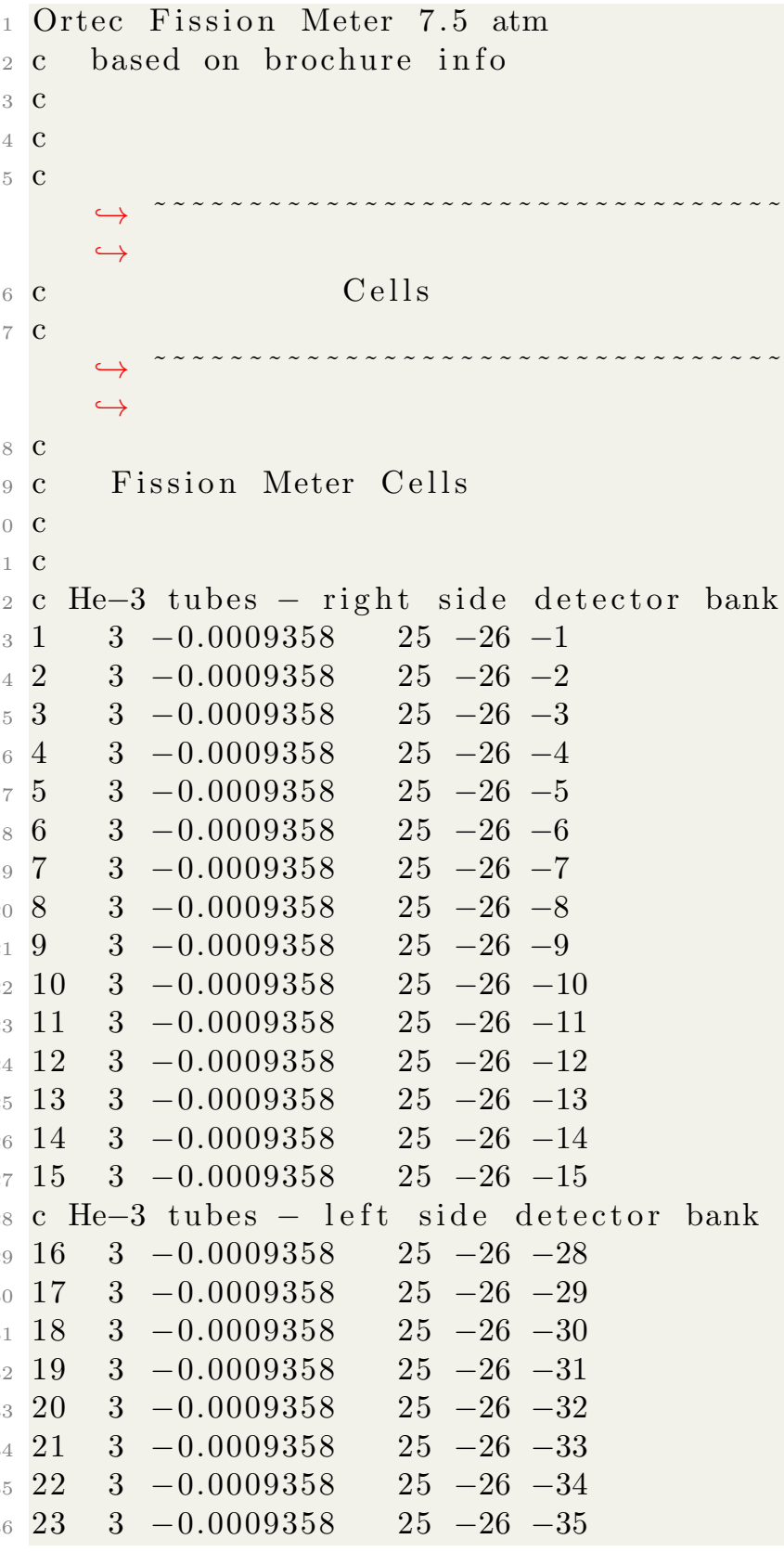




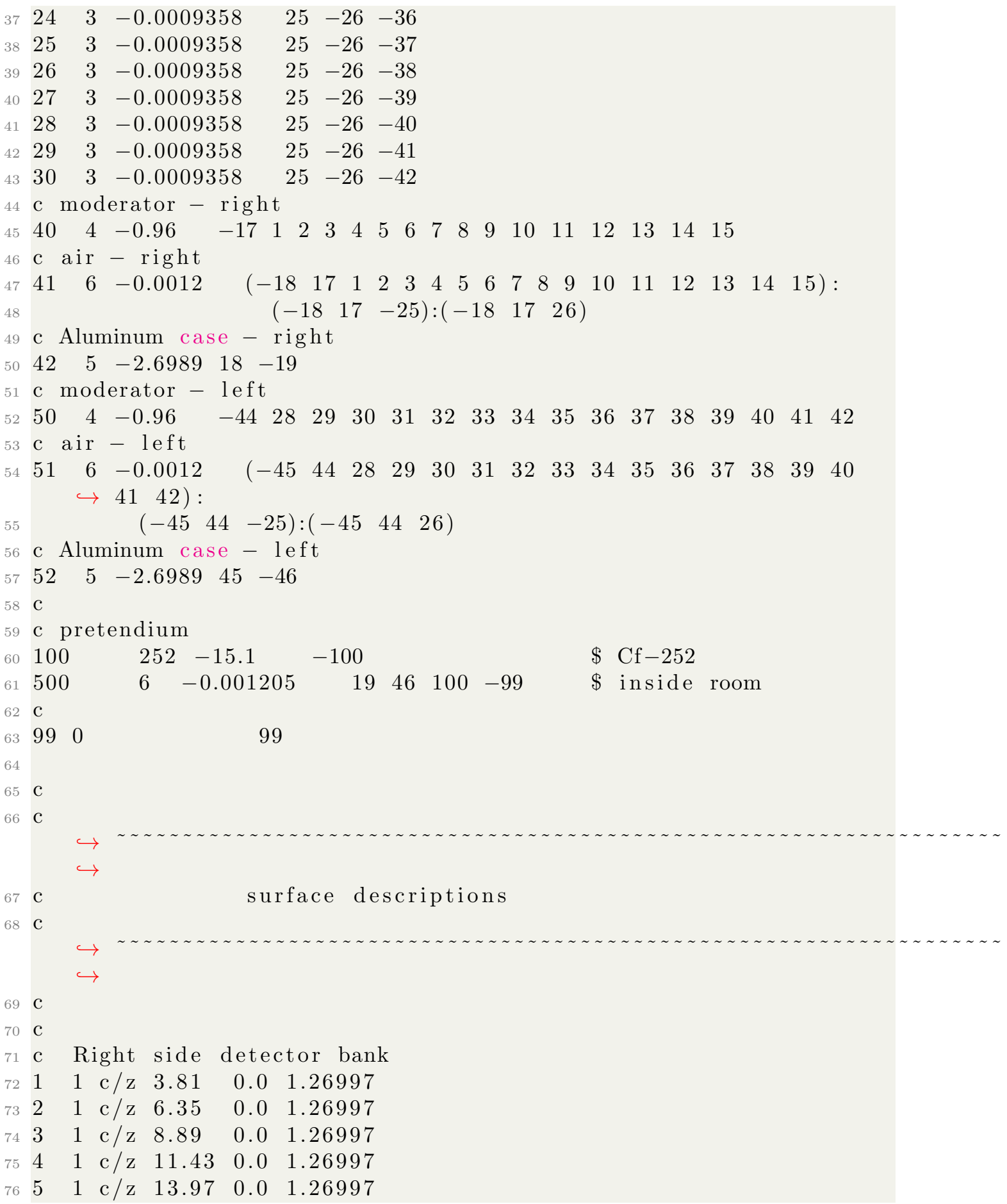




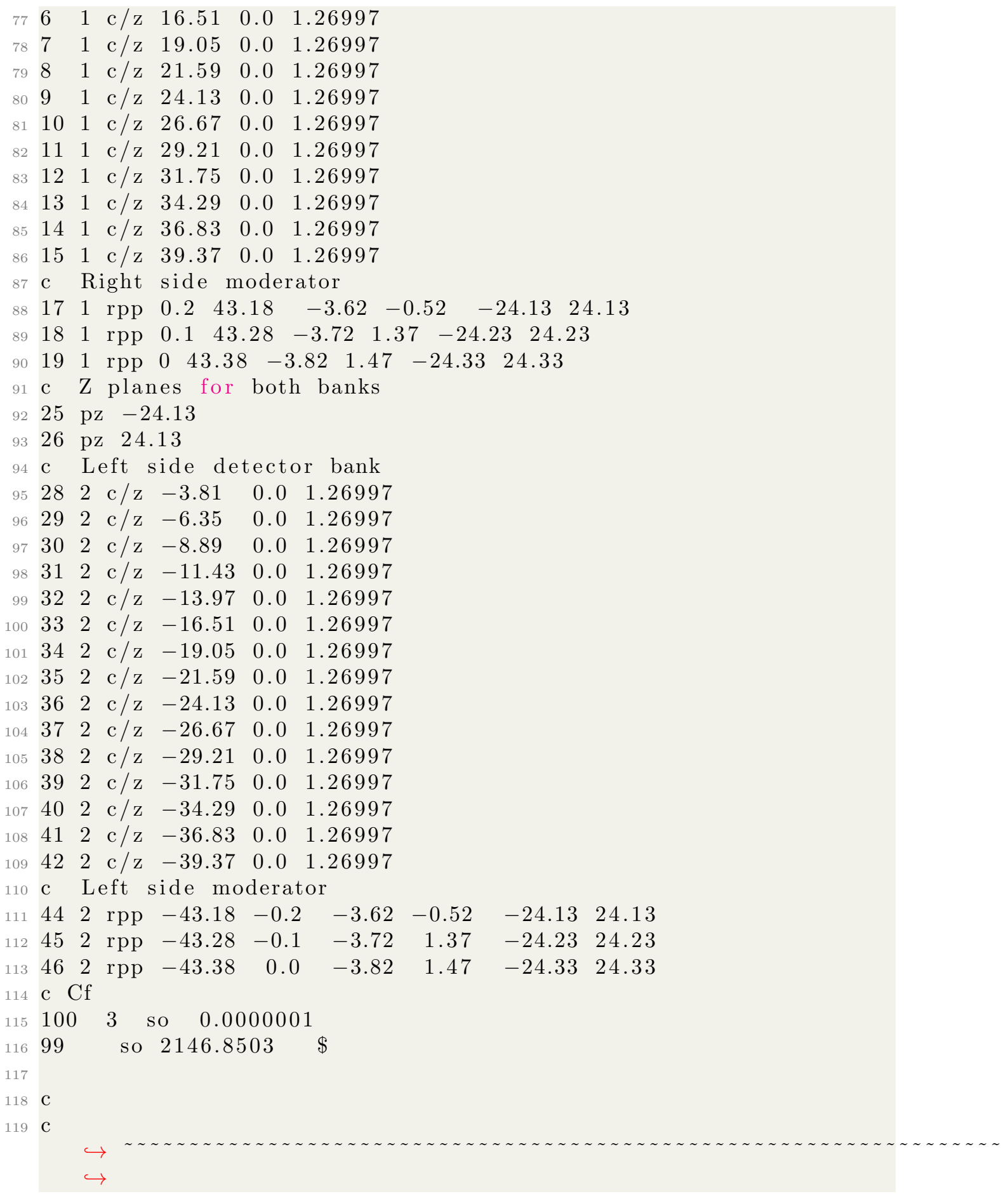




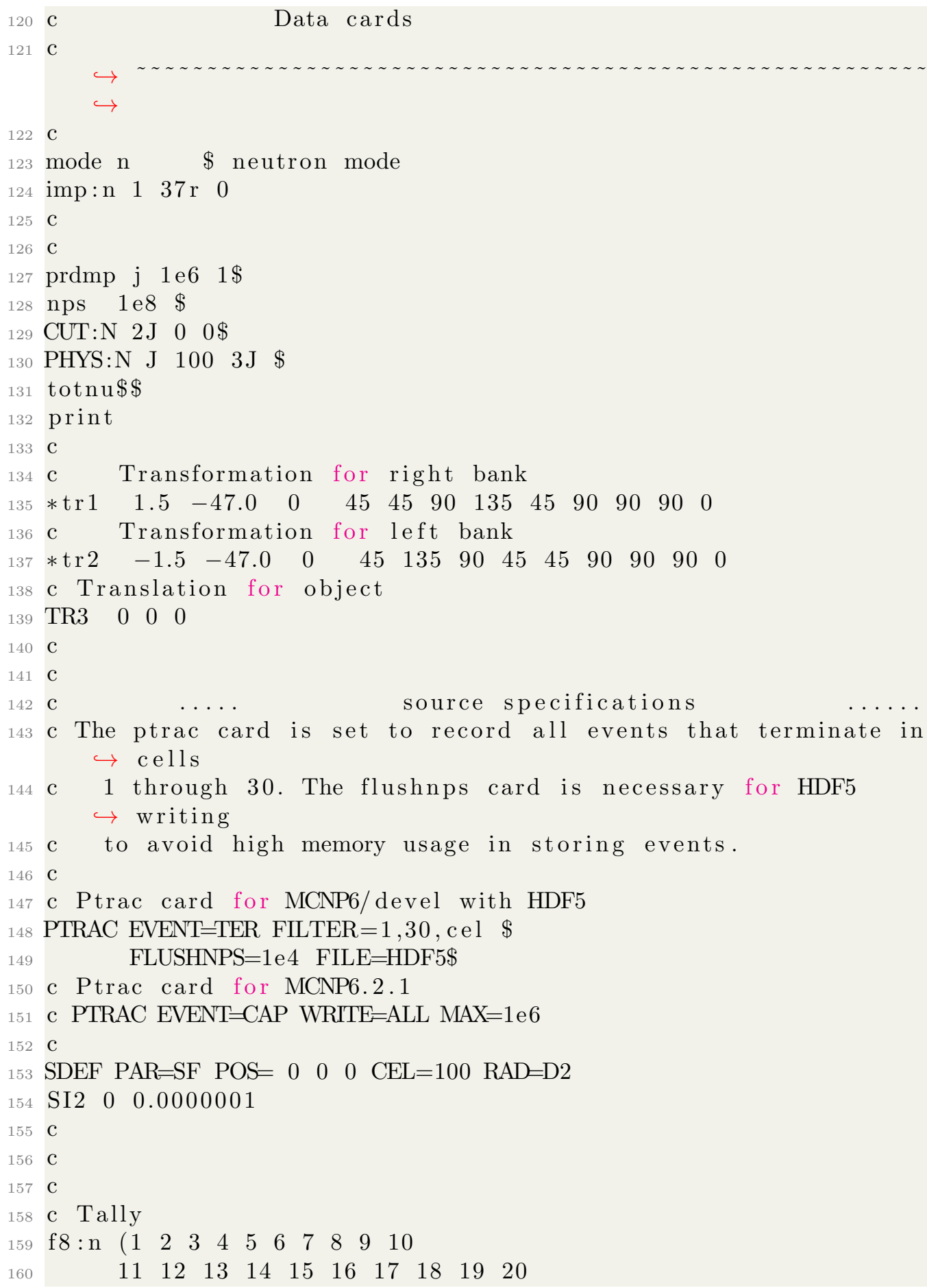




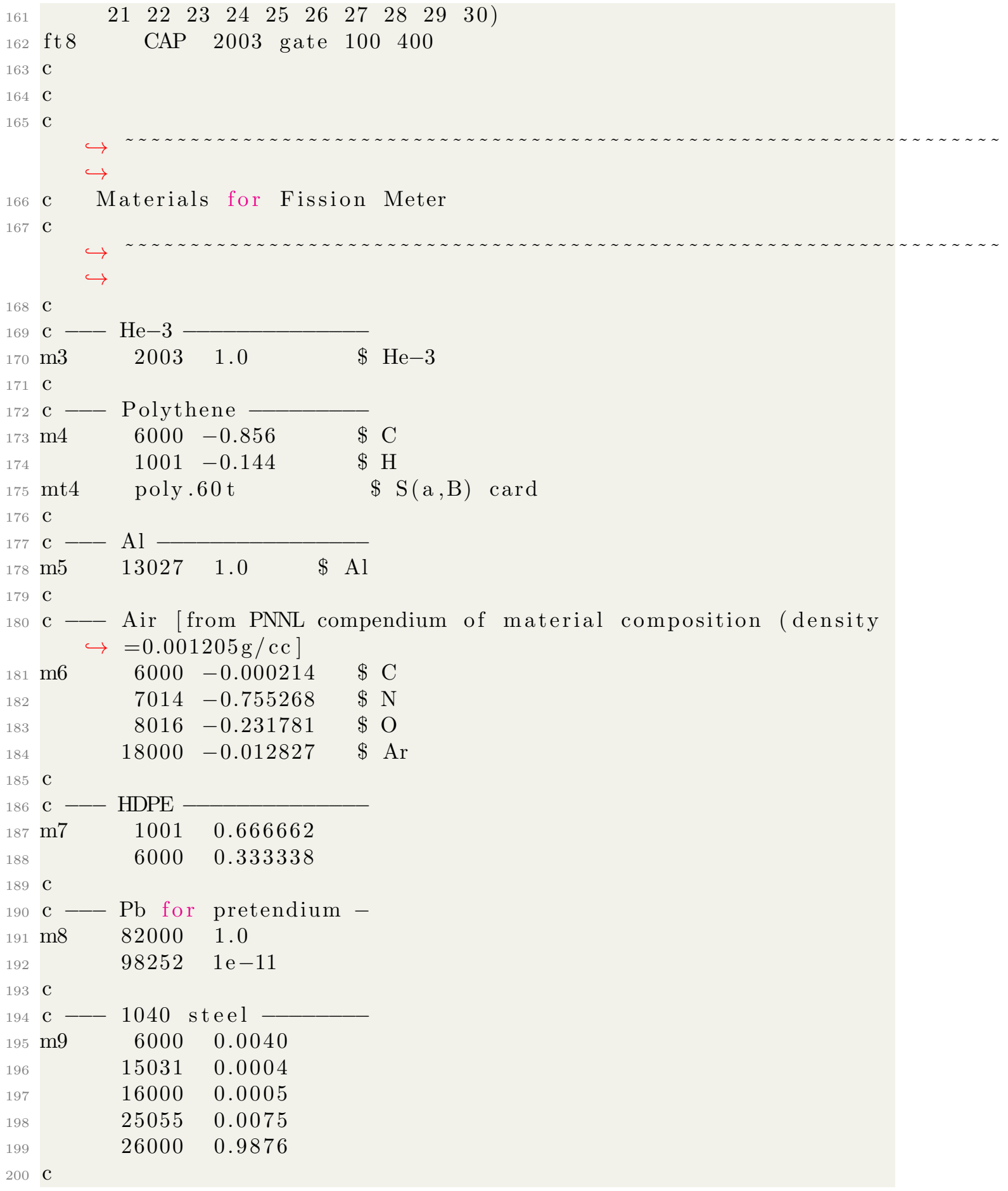




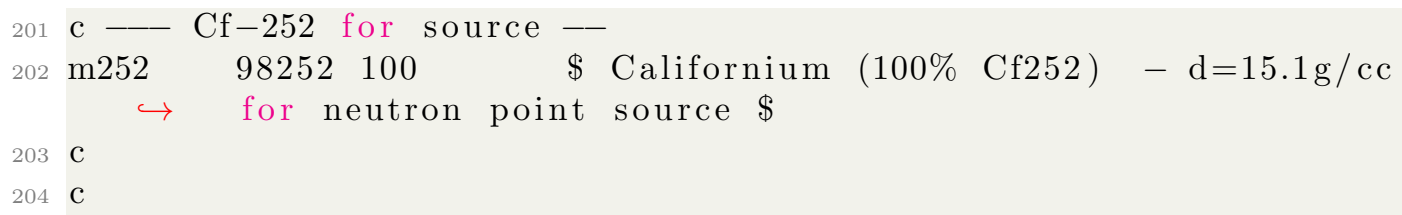




\subsection{Python script configuration: Cf-252 point source}

The configuration file for the ptrac_feynman_y.py script describes a neutron measurement with the Fission Meter of a point Cf-252 spontaneous fission source.

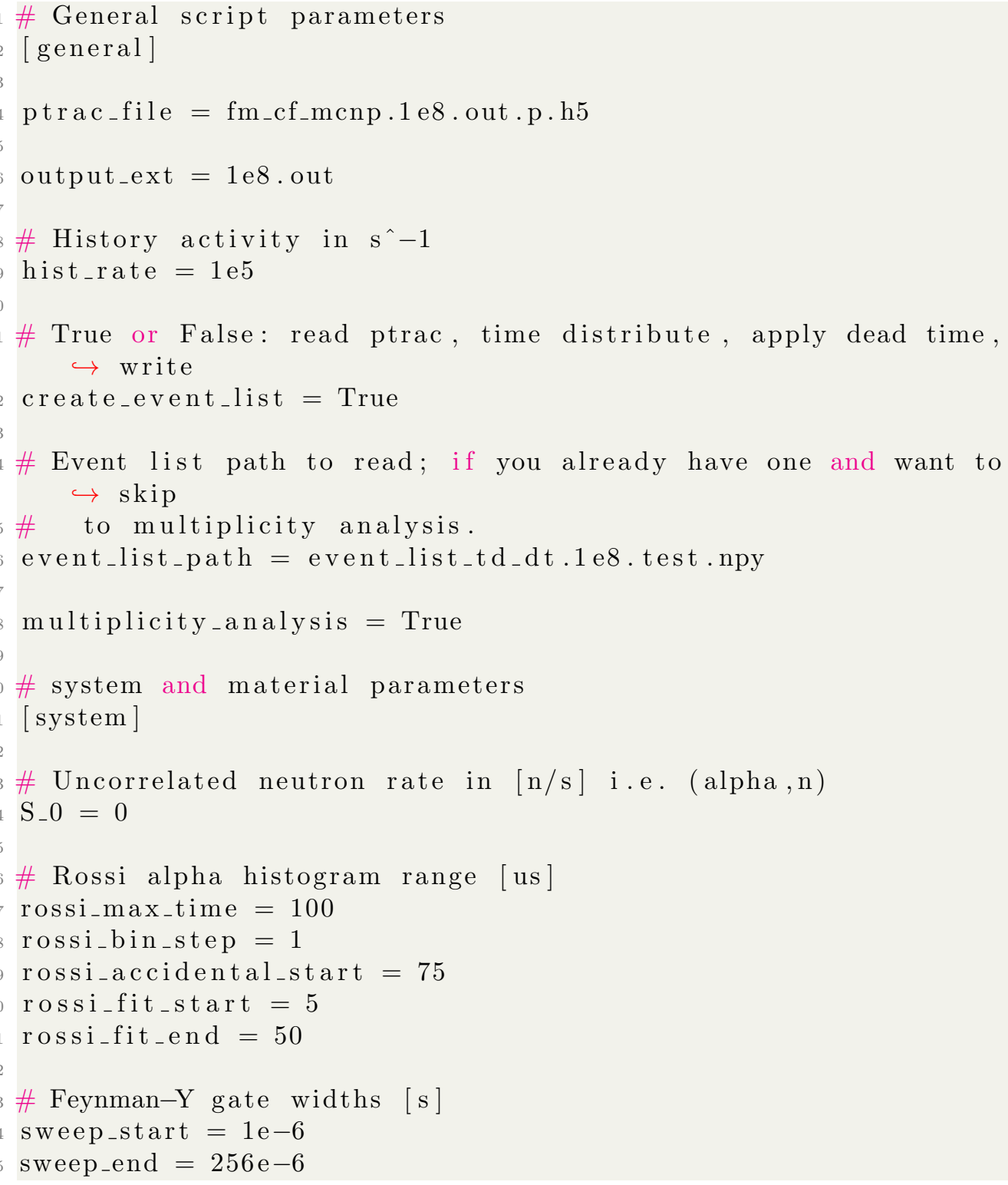




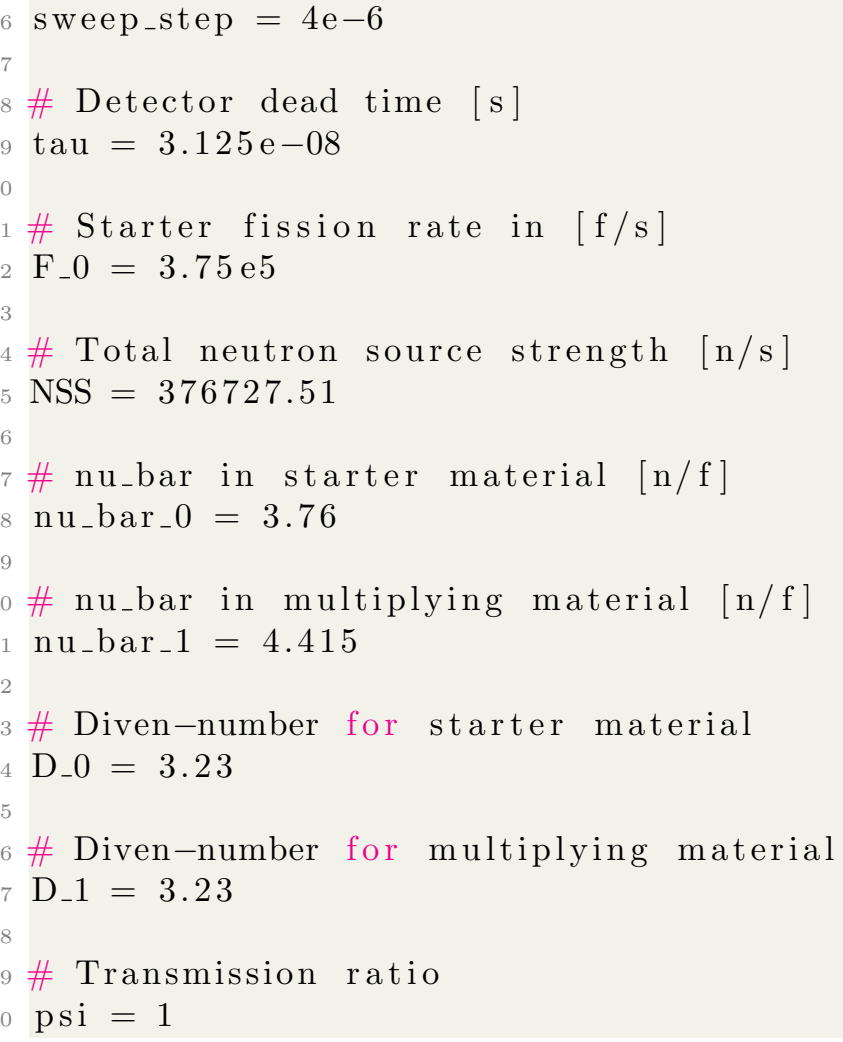




\subsection{MCNP6 model: TAI 1}

The MCNP model describes a neutron measurement with the Fission Meter of the TAI 1 source.

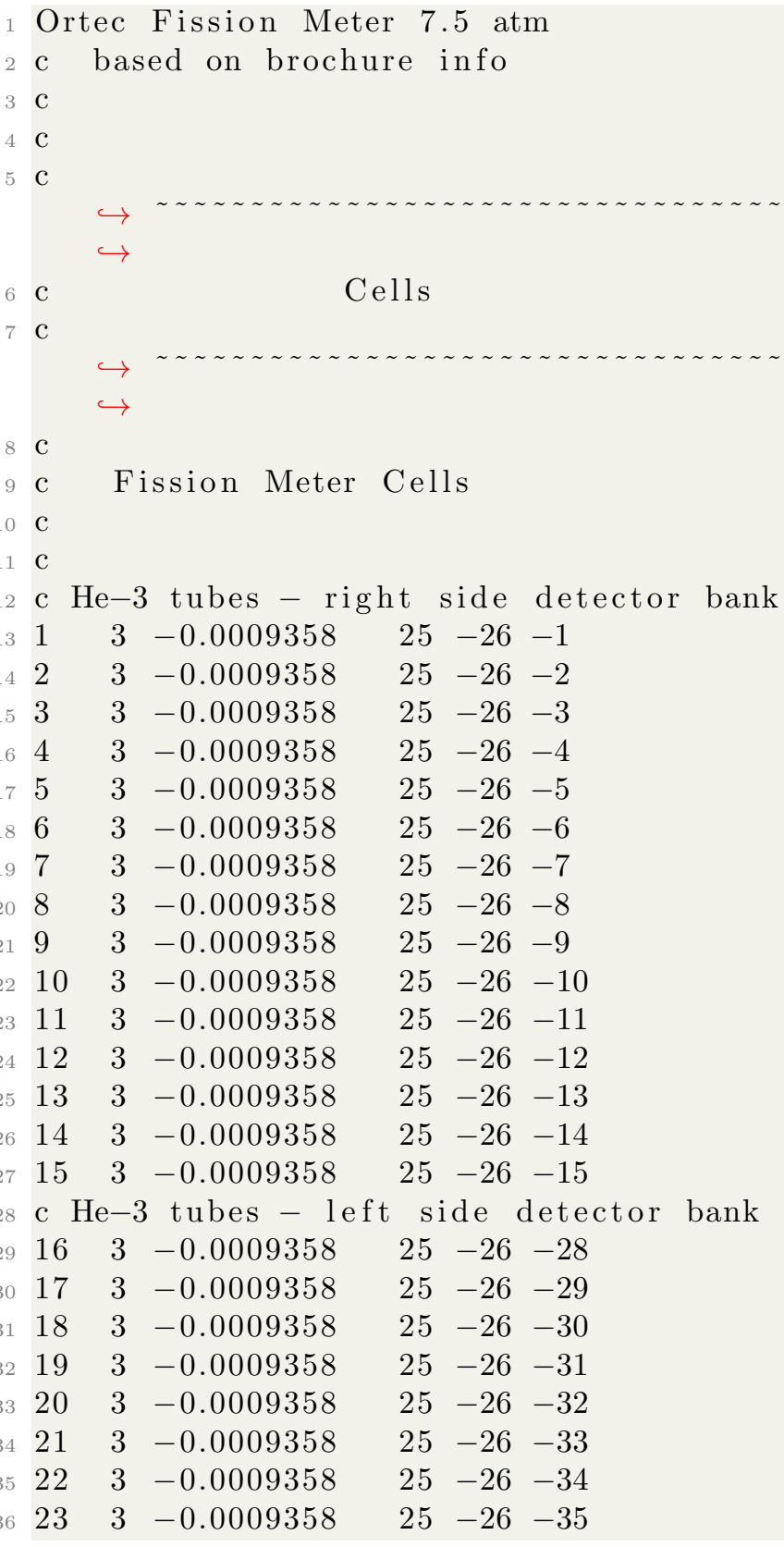




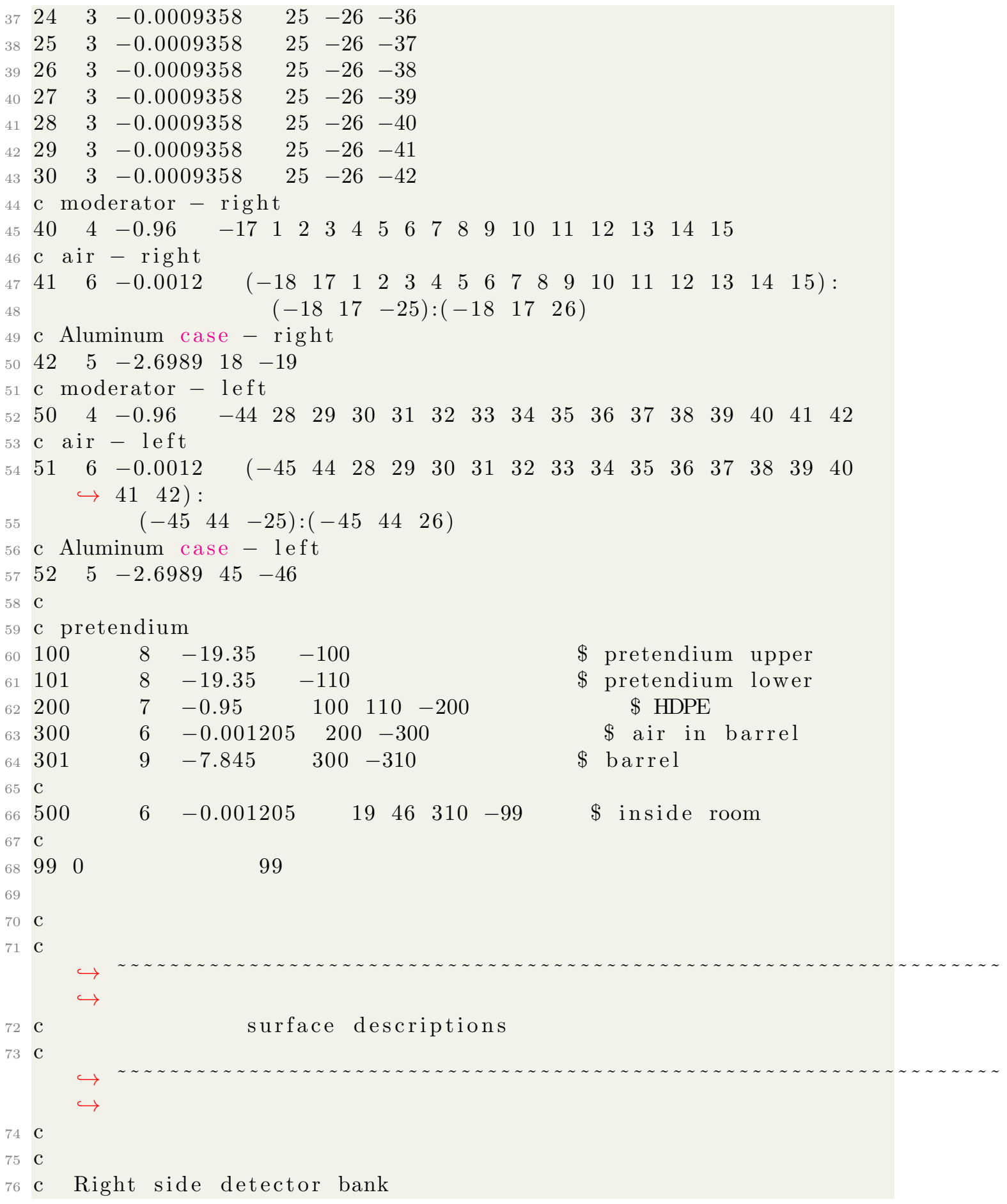




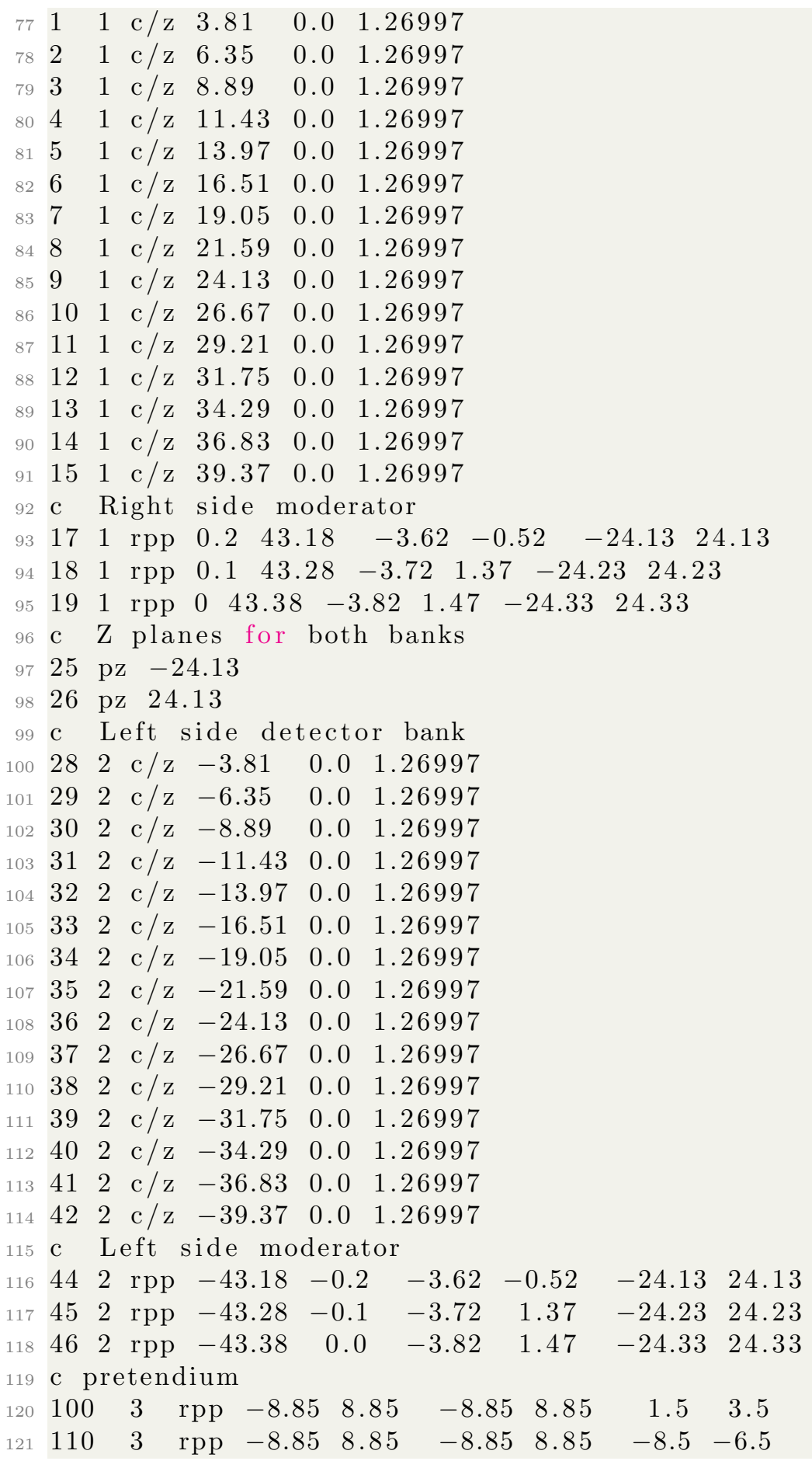




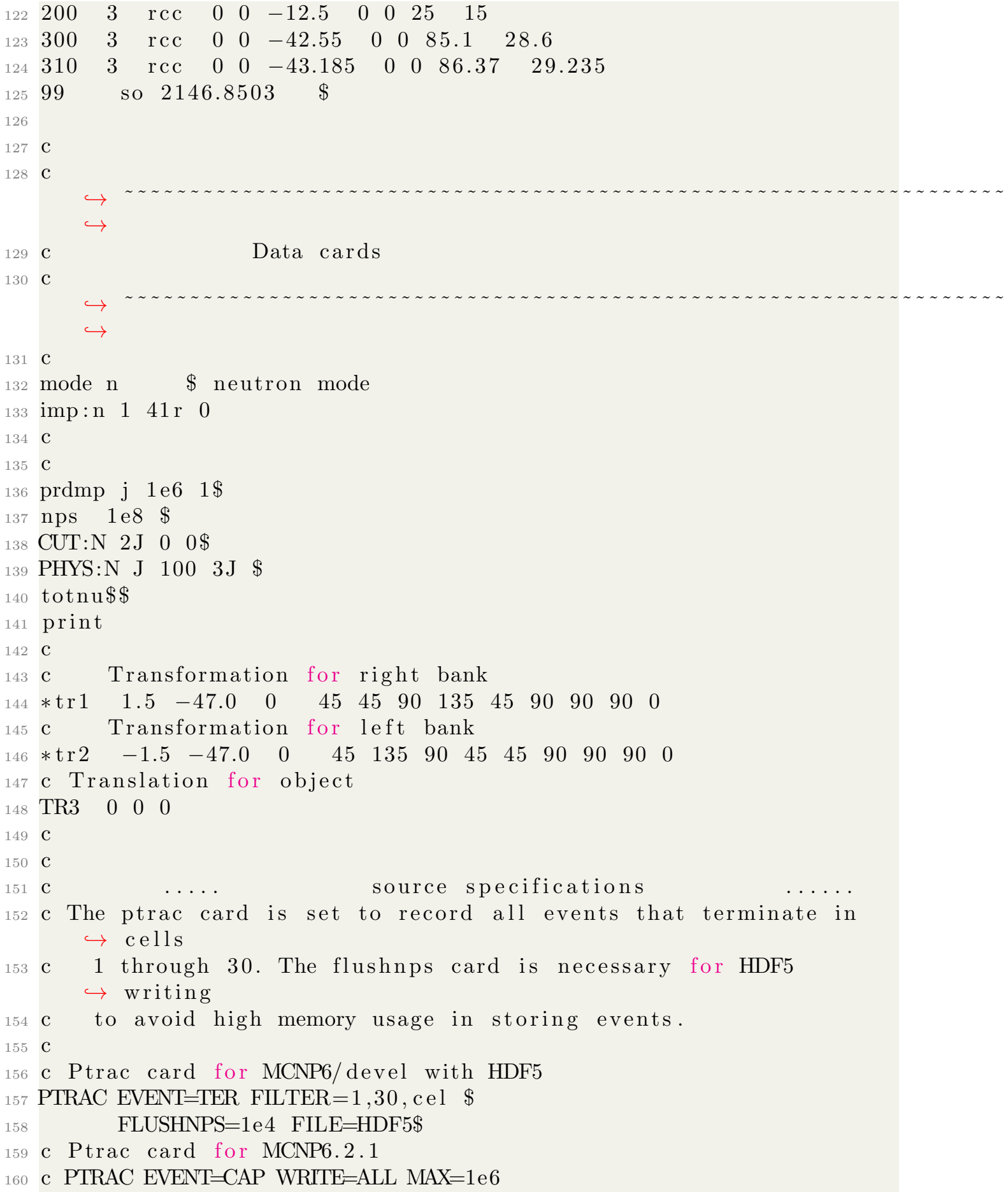




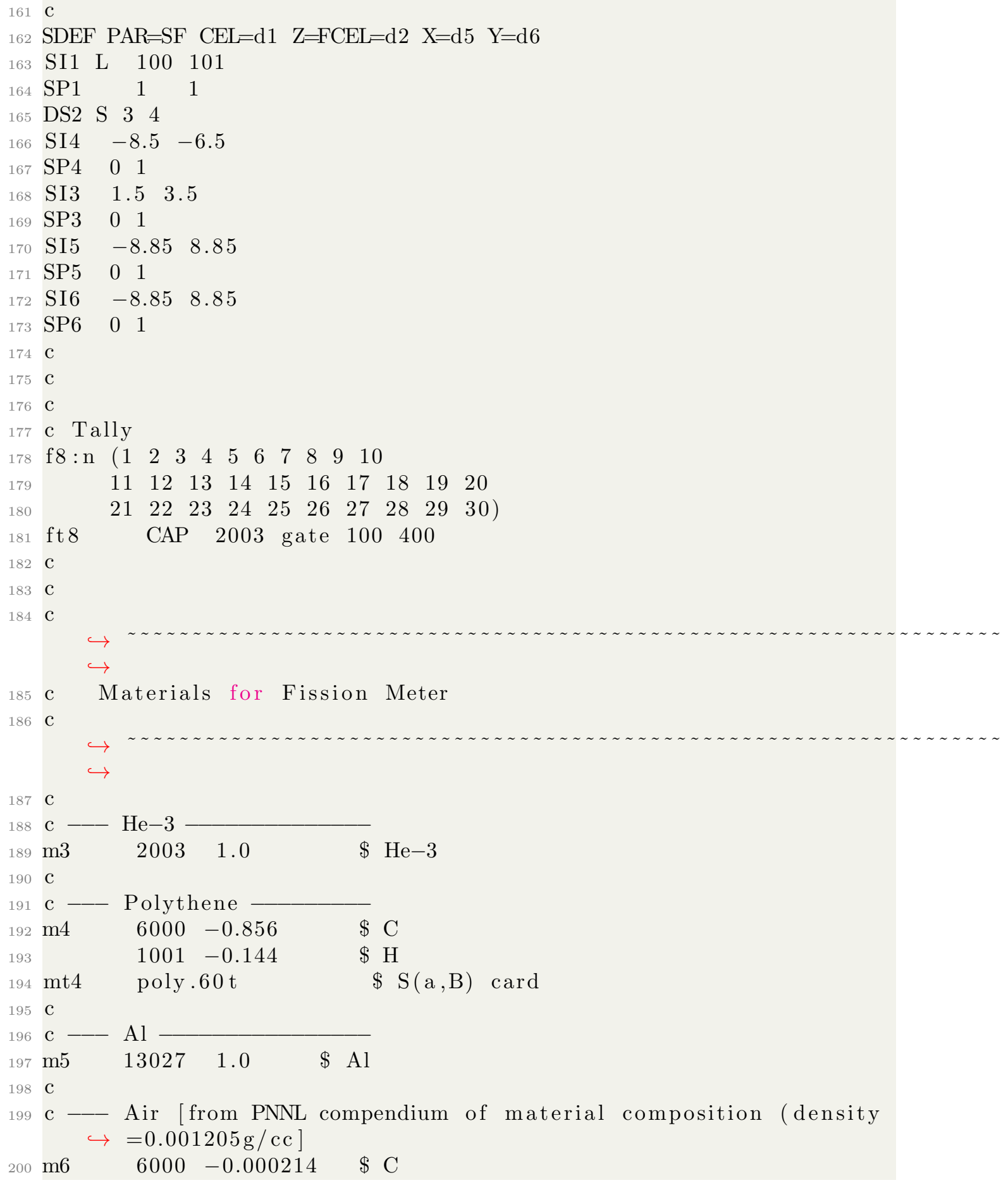




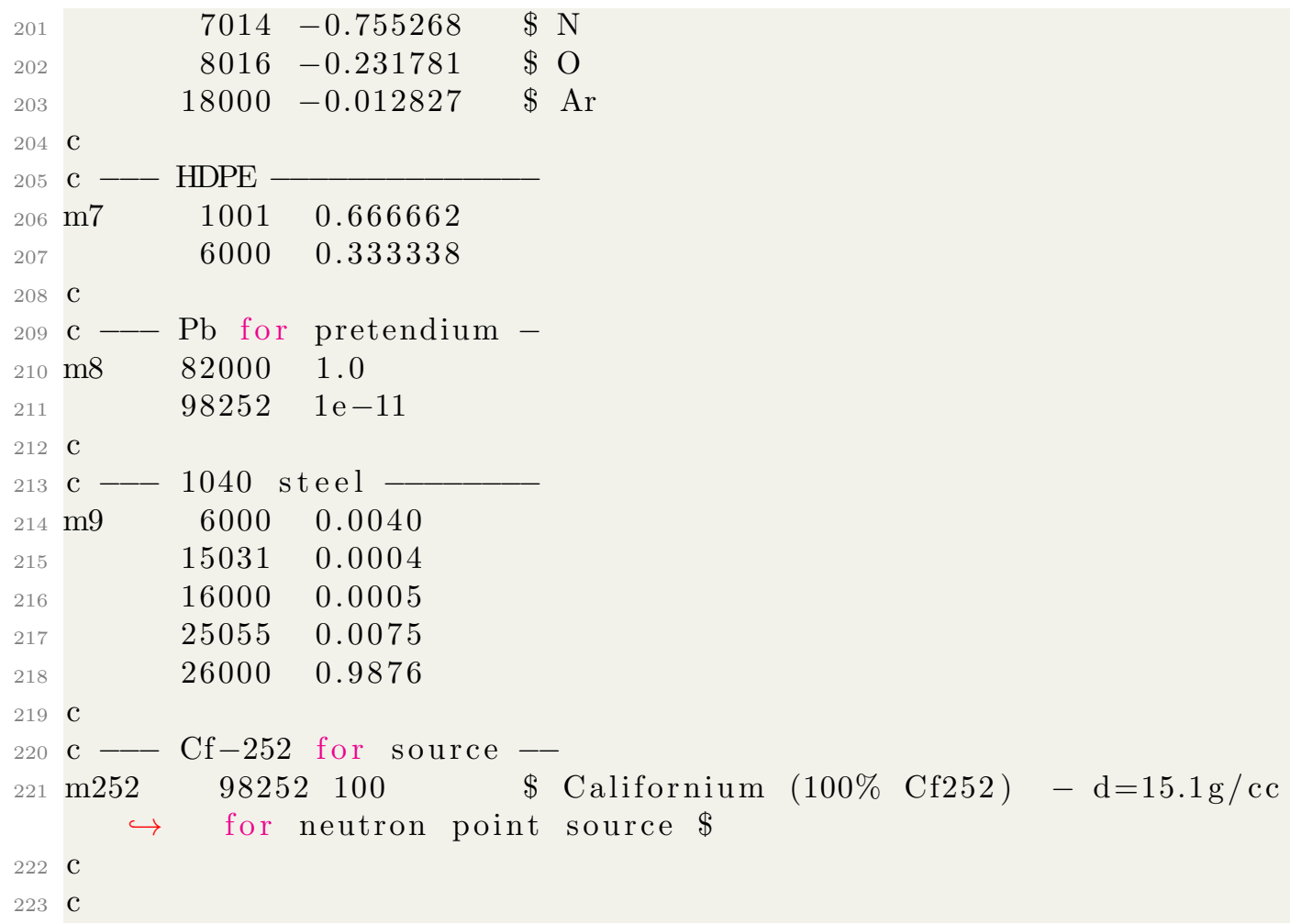




\subsection{Python script configuration: TAI 1}

The configuration file for the ptrac_feynman_y.py script describes a neutron measurement with the Fission Meter of the TAI 1 source.

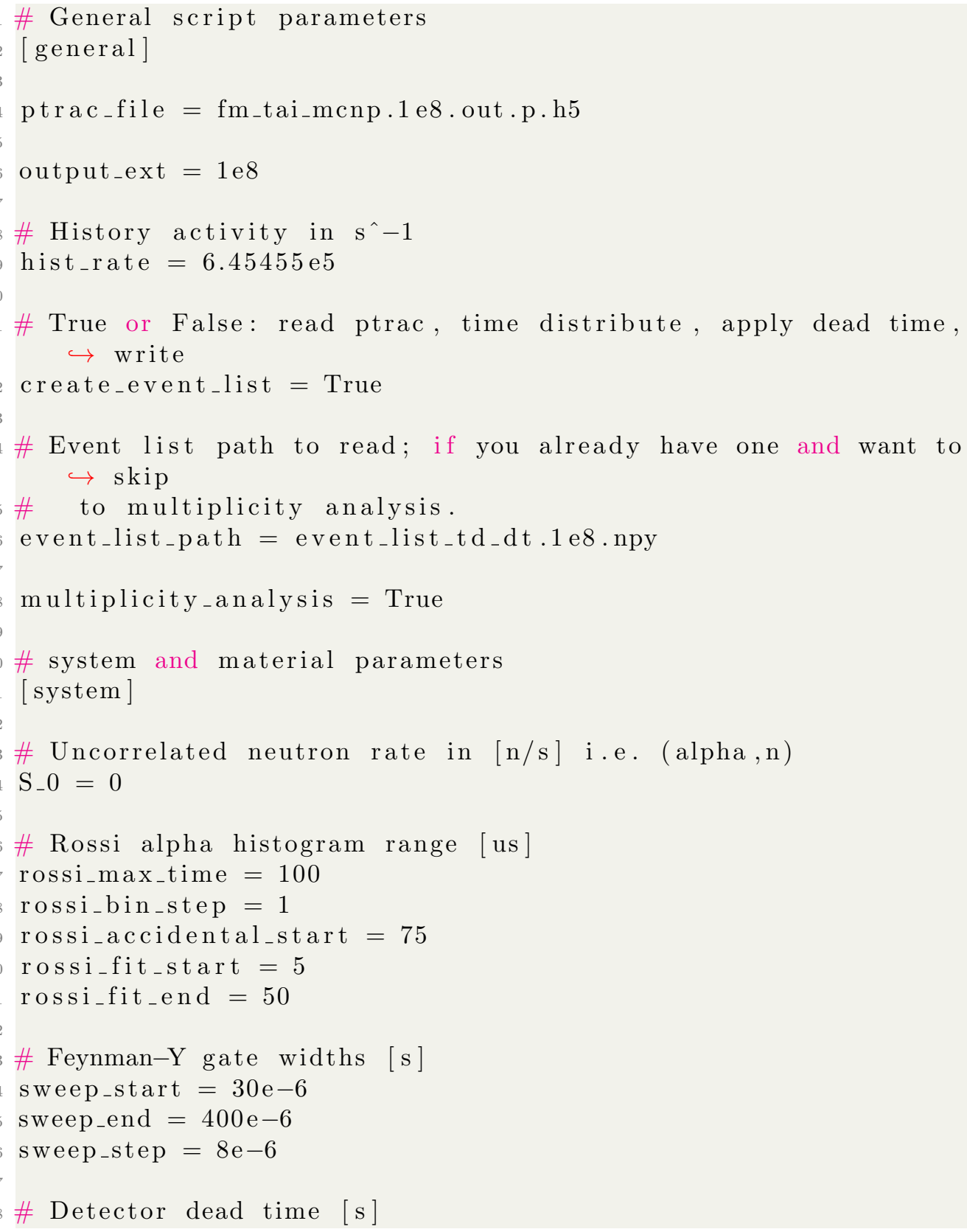




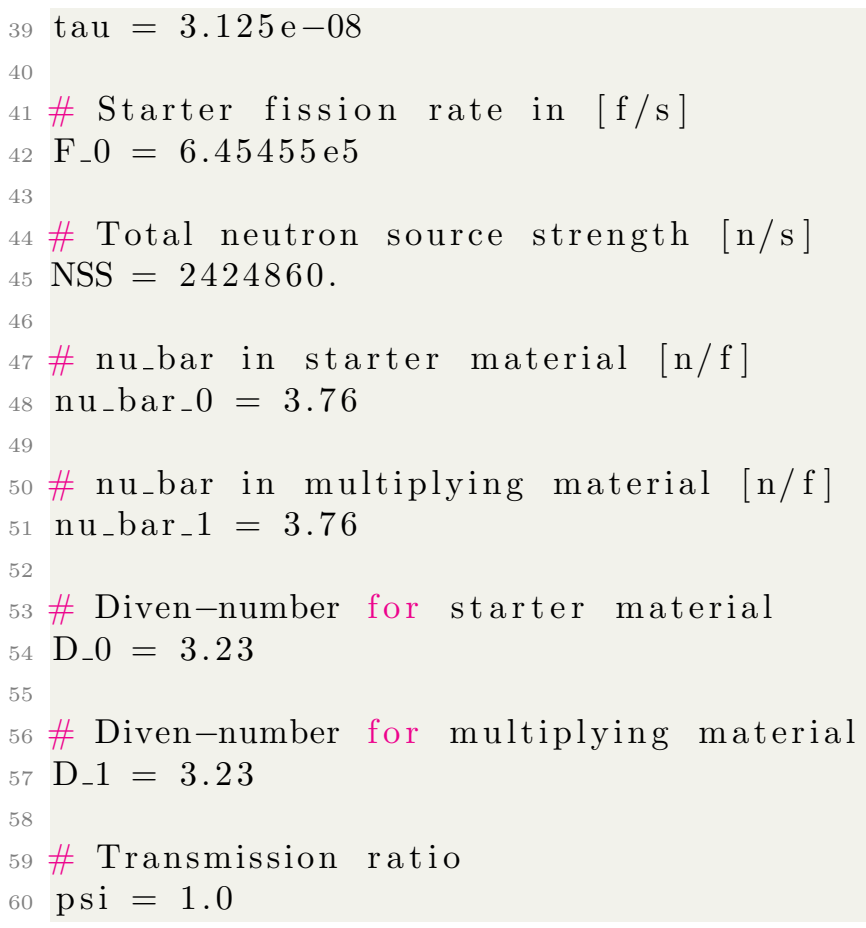




\subsection{Python results plotter}

The python script plots the ptrac_feynman_y.py Rossi-alpha and Feynman-Y outputs.

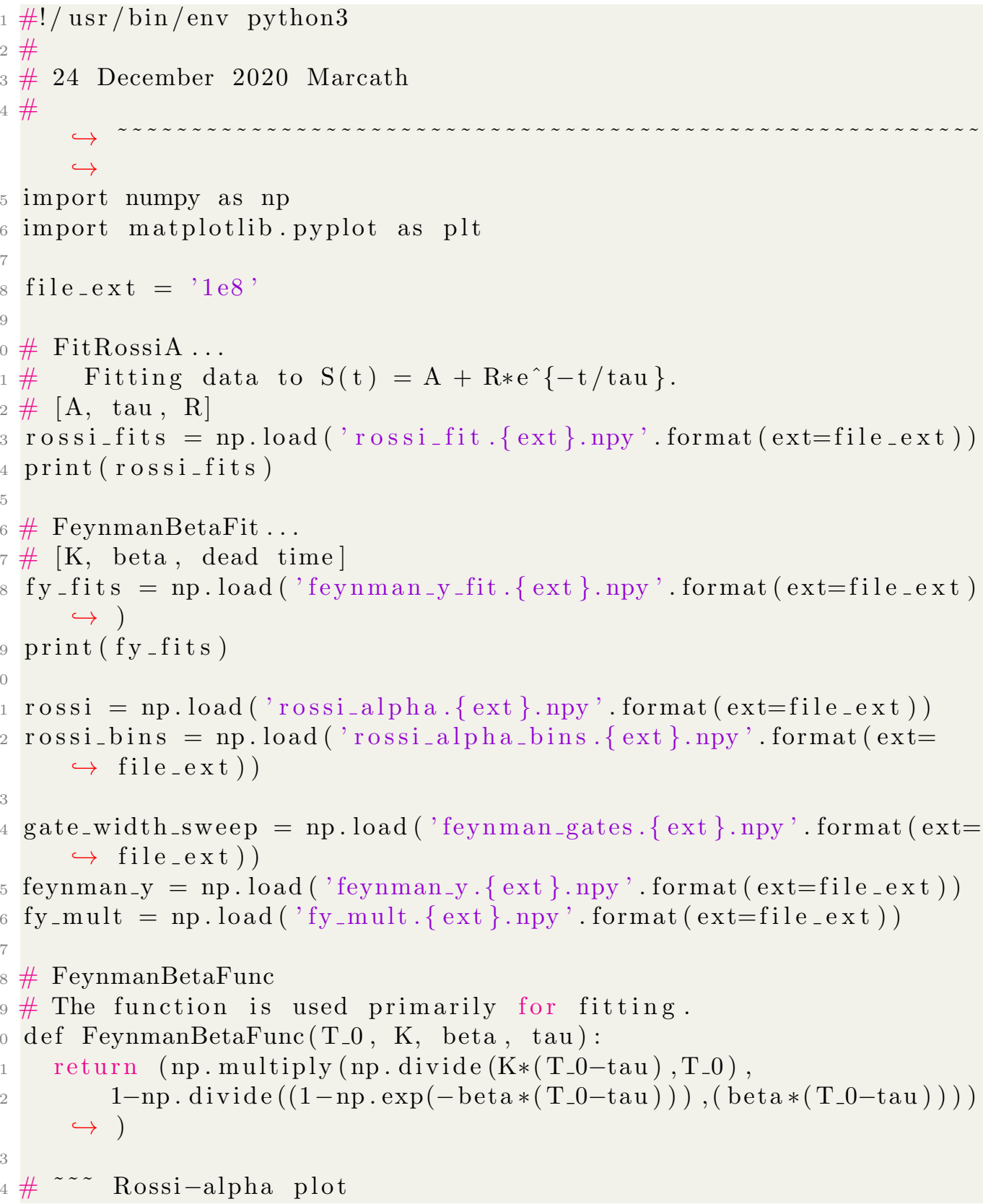




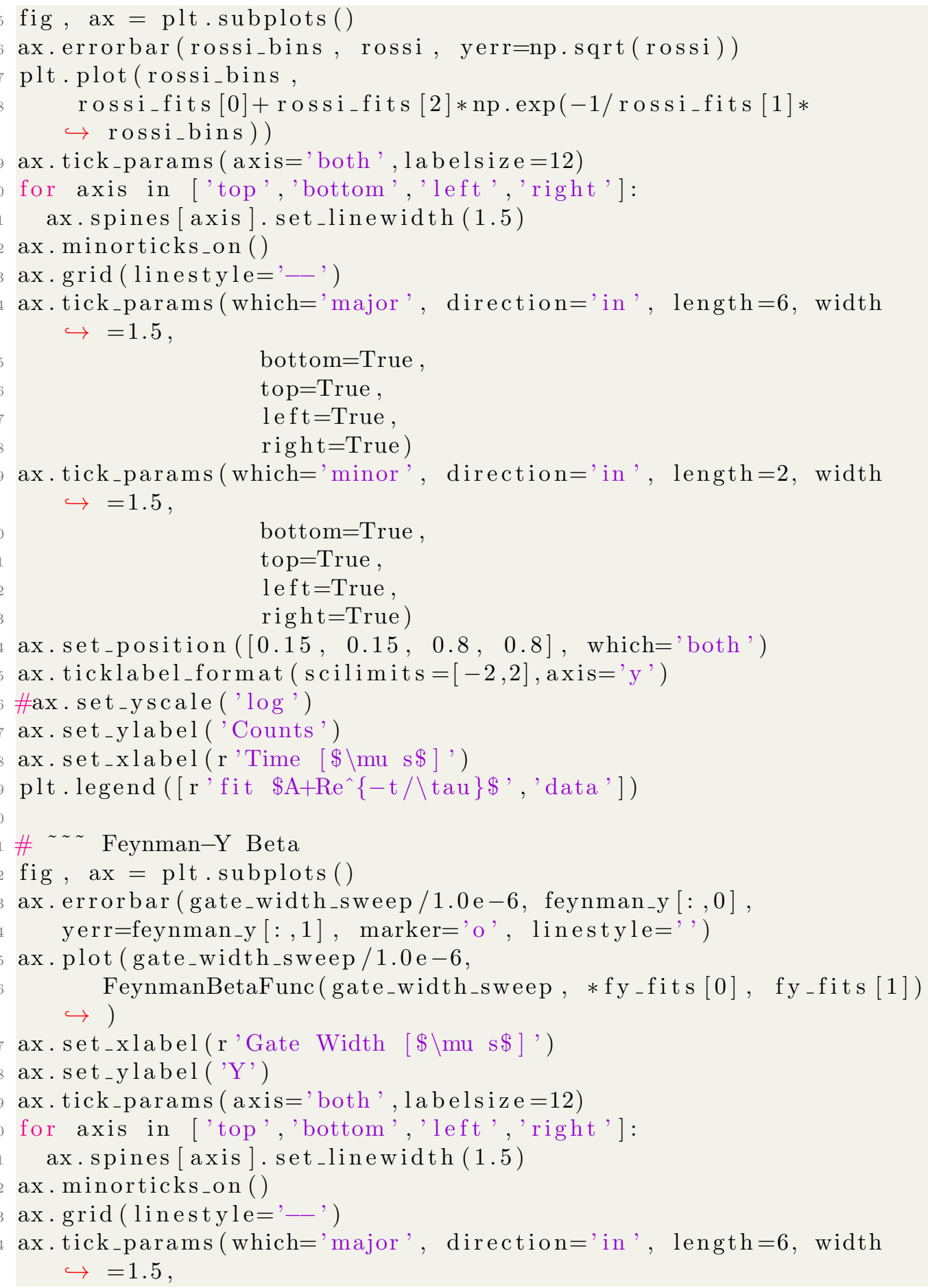




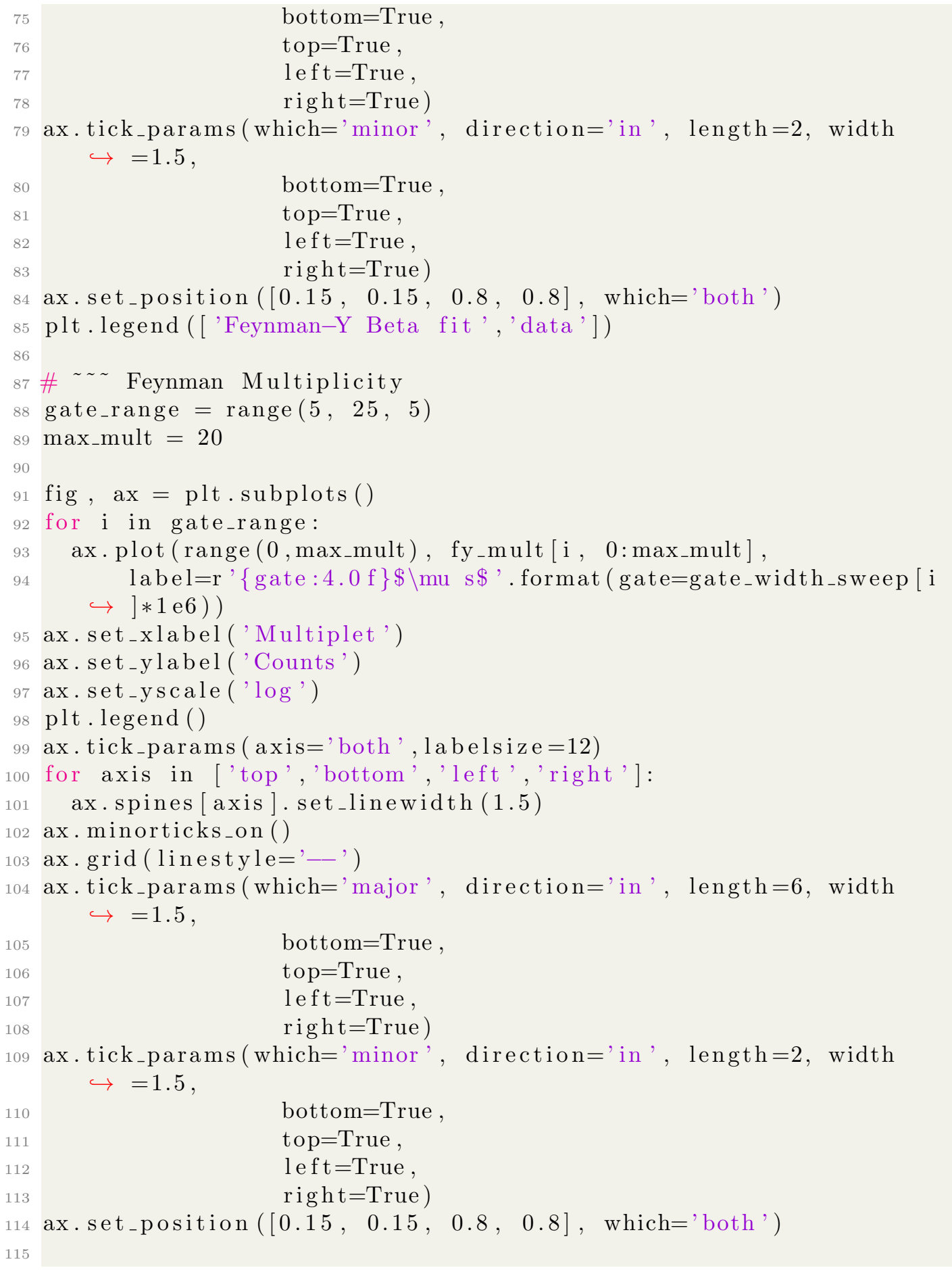


116 plt. show () 